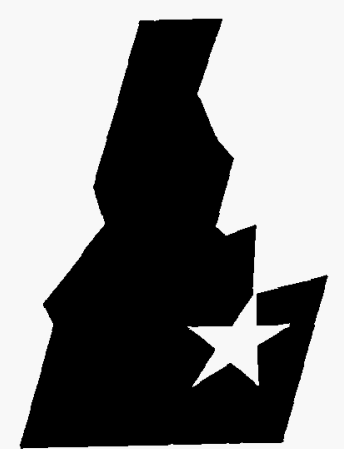

Idaho

National

Engineering Laboratory
INEL-96/0185

June 1996
RECEIVED

JUL 291996

OSTI

G. D. Lassahn

MASTER

三LLockheed

Idaho Technologles Company

DISTRIBUTION OF THIS DOCUMENT IS UNLMMED 


\title{
Automatic TLI Recognition System Beta Prototype Testing
}

\author{
G. D. Lassahn
}

HQ PROJECT MANAGER - Stephen Herrick

PROJECT NUMBER - ST474E

Published June 1996

Idaho National Engineering Laboratory

Lockheed Martin Idaho Technologies

Idaho Falls, Idaho 83415

Prepared for the

U.S. Department of Energy under DOE Idaho Field Office

Contract DE-AC07-76ID01570 


\section{DISCLAIMER}

Portions of this document may be illegible in electronic image products. Images are produced from the best available original document. 


\begin{abstract}
This report describes the beta prototype automatic target recognition system ATR3, and some performance tests done with this system. This is a fully operational system, with a high computational speed. It is useful for finding any kind of target in digitized image data, and as a general purpose image analysis tool.
\end{abstract}




\section{CONTENTS}

ABSTRACT $\ldots \ldots \ldots \ldots \ldots \ldots \ldots \ldots \ldots \ldots \ldots \ldots \ldots \ldots \ldots$ ii

INTRODUCTION $\ldots \ldots \ldots \ldots \ldots \ldots \ldots \ldots \ldots \ldots \ldots \ldots \ldots \ldots \ldots \ldots$

FUNCTIONAL DESCRIPTION $\ldots \ldots \ldots \ldots \ldots \ldots \ldots \ldots \ldots$

HARDWARE DESCRIPTION $\ldots \ldots \ldots \ldots \ldots \ldots \ldots \ldots \ldots$

SOFTWARE DESCRIPTION $\ldots \ldots \ldots \ldots \ldots \ldots \ldots \ldots \ldots \ldots$

Surveillance Program E . . . . . . . . . . . . . 8

Training Program $F \ldots \ldots \ldots \ldots \ldots \ldots \ldots \ldots$

Mask Creation Program $G \ldots \ldots \ldots \ldots \ldots$

SYSTEM TESTS $\ldots \ldots \ldots \ldots \ldots \ldots \ldots \ldots \ldots \ldots \ldots \ldots \ldots$

CONCLUSIONS $\ldots \ldots \ldots \ldots \ldots \ldots \ldots \ldots \ldots \ldots \ldots \ldots \ldots \ldots \ldots \ldots$

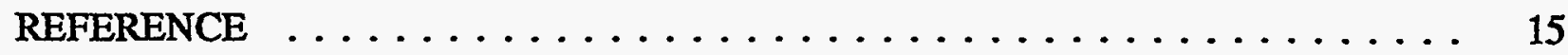




\section{Automatic TLI Recognition System Beta Prototype Testing}

\section{INTRODUCTION}

The purpose of this project is to develop both the hardware and the software for an Automatic Treaty-Limited Item Recognition system, or an automatic target recognition (ATR) system. We have tested and compared three different hardware systems ${ }^{1}$, all with the same software, and we have selected the system ATR3 as being generally preferred. This report describes the beta prototype system ATR3 (with software version 14) and some test and evaluation results.

\section{FUNCTIONAL DESCRIPTION}

This ATR system is a computer designed to find targets in digitized image data, in a very general sense. We present the ATR system with one or several images of a scene, and the ATR system tells us whether there is a target in the scene and, if there are any, where each target is. The following paragraphs describe some of the features of this ATR system:

\section{Targets}

The targets could be traditional military targets, such as airplanes or trucks. In this ATR system, the word "target" has a broader meaning: a target is anything that is potentially recognizable in the image data. A target could be, for example, a physical object, like an airplane or a golf ball or a river; a structure such as a crack in a surface or a hot spot detected by an imaging heat sensor; an area of chemical contamination on the ground; a particular combination of types of vegetation growing in an area; a certain type of terrain, such as gently rolling hills; or any other pattern that is distinguishable in the image data.

\section{Images}

By "image" data, we mean an array of digital data values versus position in two dimensions. A standard digitized video picture, with brightness versus $\mathrm{x}$ and $\mathrm{y}$ coordinates, is such an image. Instead of brightness, the image data for this ATR system could be any quantity such as temperature, ground surface elevation, ultrasonic reflectivity, eddy current readings, radar range readings, Raman scattering intensity at a particular wavelength, optical emissivity, mechanical strain, etc. The only requirement is that the data be in the form of a two-dimensional array of values.

\section{Image Data Fusion}

This ATR system is designed to produce one result ("target" or "no target") from the simultaneous analysis of several images comprising different types of data, such as radar reflectivity, infrared emission, and surface altitude, all versus $x$ and $y$ position, all for the same scene. That is, this ATR system uses image data fusion. The presumption is that a target can be recognized much more reliably if we have several different types of image data instead of just 
one type. However, this ATR system is still fully functional with as few as one image per scene.

\section{Uncertainty Estimation}

No one expects that any ATR system will be perfect at recognizing targets. This ATR system tells us about its own imperfections and limitations for any application, by giving an uncertainty estimate. That is, this ATR system tells us how well it can do at recognizing the specified type of target in the given type of data. In some applications, the given data is simply not adequate to distinguish between the specified targets and background. This ATR system's uncertainty estimate is actually a prediction of the rate of errors in target recognition, a prediction of the fraction of pixels that this ATR system will incorrectly call "target" or "background". The uncertainty is very strongly dependent on the specific application, and it is estimated separately for each application.

\section{Error Minimization}

An ATR system can make two types of errors: incorrectly calling a background region a target, or incorrectly calling a target background. The rate of either type of error can be made as small as we would like, if we are willing to tolerate a very large rate for the other type of error. In practice, we choose a coefficient indicating the relative importance of the two types of error. Normally, we say that it is much more important to not miss any targets than to not have any false indications of targets. This approach can make this ATR system a very effective screening tool: it can be used to discard scenes that definitely do not include targets, and to flag for expert human inspection those scenes that might possibly contain a target. Such an approach can greatly reduce the amount of human labor involved in searching for targets, without any significant risk of missing targets.

\section{Training Process}

This ATR system uses a training process. For training, an operator gives the ATR system a set of data in which the operator has marked some regions of some scenes as targets and some other regions as background (that is, definitely not targets). Some regions of the training scenes may be left un-marked, and these regions are ignored by the training process. The training data set should be similar in character to the surveillance data that will be analyzed later, having both similar targets and similar background regions. Normally, better results are expected if the training data set is large, including many scenes with many (tens or hundreds) targets and many background regions marked by the operator.

This training process defines what a target is, by giving the ATR system examples of targets. It also gives the uncertainty estimate for this application. It also tells us the relative importance of each of the different types of image data given to the ATR system, so that we have the option of simplifying the overall process by not bothering with unimportant measurements (if there are any). Most importantly, the training processes gives values of coefficients to be used for optimal processing of surveillance data.

\section{Surveillance Data Processing}

After the training process is finished, this ATR system is used in a production mode, in which it simply looks at the image data for a scene and tells us whether there is a target in that scene. Using this mode to process surveillance data is very simple, requiring only that the operator tell the program the names of the computer files containing the image data. There are 
several options for displaying the ATR system's results to the operator, including one as simple as printing "YES" or "NO" on the computer display, and another in which the scene is displayed with color coding to show the locations of targets.

\section{Ease of Use}

In the production mode, for processing surveillance data, this ATR system is very easy to use, requiring only very basic knowledge of how to operate a personal computer.

In the training process, certain expertise is required. A skilled operator must give the ATR system a list of local features to be extracted from the images and used in recognizing targets. These features might be such things as a measurement magnitude, smoothness of a measurement versus position, the presence of a step change in a measurement versus position, and a multitude of other local structures in the measurement versus position maps. The ATR system will select the important features from the operator-supplied list, and in effect ignore the unimportant features, so it does not matter. if the operator specifies superfluous features. However, the ATR system cannot invent its own features, so the operator must supply a feature list that includes enough features to distinguish between target and background. This can require some understanding of the nature of the targets and of the measurement systems. Note, however, that the operator only has to supply the ATR system with a sufficient set of features, a set of tools that will enable target-background discrimination. The operator does not necessarily have to understand how these tools will be used, or exactly how a target will be recognized, or even which tools will be important. This allows the ATR system to effectively implement target recognition procedures that are very complicated, much too complicated to be used in practice by even expert data analysts.

\section{Size of Data Sets}

This ATR system analyzes one scene at a time, so there is no practical limit on the number of scenes that can be processed. The number of images per scene, and the number of pixels (sample points or measurement points) per image, are limited only by the memory in the parallel processor nodes, which is $\mathbf{8 0}$ megabytes in ATR3. In the event that this memory is inadequate for a particular application, this ATR system can analyze each scene in parts, effectively dividing each scene into several smaller scenes, and thereby circumvent any memory limitations.

\section{System Hardware}

The unique aspect of this ATR hardware is the use of small, low-cost parallel processors to give a lot of computing speed in a small, cheap package. It is easy to add more processors to this type of system and thereby increase the speed, for most operations, proportionately. The parallel processors for this beta prototype system ATR3 are Alta Technology AL/V66 processors, and the host computer is a DEC 2000 AXP. This system hardware is described in more detail later in this report.

\section{System Software}

This ATR system does use such basic, standard software as the operating system normally supplied with the host computer and a host-network interface normally supplied with the parallel processor network. Other than this, all the software was developed by the Idaho National Engineering Laboratory for the Department of Energy, so it has no licensing restrictions. The 
source code can easily be modified to include additional functions or non-standard image manipulations. The software is discussed in more detail in a later section of this report.

\section{Other Uses}

As with any automatic target recognition system, this ATR software includes a lot of general-purpose image analysis capability, and the system can easily be used for image manipulations other than target searches.

The presence of the ATR-related hardware and software does not prevent the host computer from being used for any other normal application, such as running other data analysis programs, word processing, spread sheets, etc. Other software and hardware (within the normal limits) can be used freely on this ATR system computer.

\section{Summary}

We have developed an Automatic Target Recognition system designed for rapid screening of large amounts of multi-image data. This system is versatile and broadly applicable, and capable of implementing very subtle and complicated target recognition procedures. It incorporates optimal image data fusion and uncertainty estimation. It is reasonably low cost, fast, and not subject to license restrictions. The software is easily expandable to incorporate new capabilities, and the hardware is easily expandable to increase the speed.

\section{HARDWARE DESCRIPTION}

The ATR3 hardware comprises two major parts: the host computer, and a daisy chain of five parallel processor nodes. The bulk of the computation is done by the parallel processors, and the calculation speed of the system can easily be increased by simply adding more nodes to the chain. Adding more nodes also increases the total memory of the system, since each node has its own memory; the nodes do not share memory. This arrangement allows the user to easily tailor the ATR hardware system to suit his own calculation speed requirements and budget constraints.

The host computer is the interface between the parallel processor network and the peripheral devices including the operator's console and any data storage and display devices. The ATR3 host computer is a DEC 2000 Model 300 AXP. A VT510 monochrome monitor and keyboard, connected to a serial port, are used as the operator's console. The high-resolution RGB monitor, with the ATR software in its normal mode, is used solely for displaying the images being processed. When the ATR software is being debugged with TCP/IP support, the RGB monitor us used with the second keyboard and the mouse to manipulate and observe the parallel processor nodes.

Each ATR3 parallel processor node is an Alta Technology AL/V66 which includes a DEC Alpha 21066 processor and 16 Mbyte of memory, Each node also includes a T425 transputer to facilitate inter-node communication and control operations such as booting the network, but the transputer's operation is transparent and the user does not need to explicitly address it. The nodes can communicate via the standard four $20 \mathrm{Mbit} / \mathrm{second}$ (about $1.8 \mathrm{Mbyte} / \mathrm{second}$ in one direction) transputer links, or T-links, and also via four $250 \mathrm{Mbit} / \mathrm{second}$ (at least 17 Mbyte/second) A-links. The parallel processor nodes can be accessed using the TCP/IP model, in which case each node's T-links 2 and 3, and one host T-link, are used by the system and are not available explicitly to the user; the other T-links and the A-links are available at the user's 
discretion. This TCP/IP access if very useful for debugging the software that runs on the parallel processor nodes, as it allows each node to be monitored in a separate window of the user's display. The parallel processor network can also be used, more simply and efficiently, without TCP/TP support, in which case all the links are at the user's disposal. The parallel processor nodes are mounted in a VME chassis, separate from the host. The ATR3 vendor recommends leaving one or two vacant slots between nodes in the VME chassis.

For historical reasons, three of the five nodes in this particular system have a clock speed of $166 \mathrm{MHz}$, and two run at $233 \mathrm{MHz}$. The current version of the ATR software cannot allocate computation tasks to the nodes differently depending on their different speeds. Therefore, the system speeds that are discussed later are determined by the speed of the $166 \mathrm{MHz}$ nodes. A system using all $233 \mathrm{MHz}$ nodes would presumably be proportionately faster.

Figure 1 indicates schematically the ATR3 hardware system. The present system does not include A-link connectors on the host; these will be supplied later by the vendor, as specified by the original purchase agreement. The details of the inter-node connections are indicated in Figure 2. The node T2 and T3 connections are used for TCP/IP support. The ATR software uses either $\mathrm{T} 0$ and $\mathrm{T} 1$ or $\mathrm{A} 0$ and $\mathrm{A} 1$ for inter-node communication. (T-links cannot be connected to A-links.) The UP and DOWN connections are always required for system operation. It would be possible to use several links in parallel; for example, we could connect the $\mathrm{A} 2$ and $\mathrm{A} 3$ links just as the $\mathrm{A} 0$ and $\mathrm{A} 1$ links are connected, and thereby double the potential inter-node data transfer rate. However, this would substantially complicate the inter-node communication part of the ATR software, and the overall ATR software is such that it could not significantly benefit from the additional parallel links. 


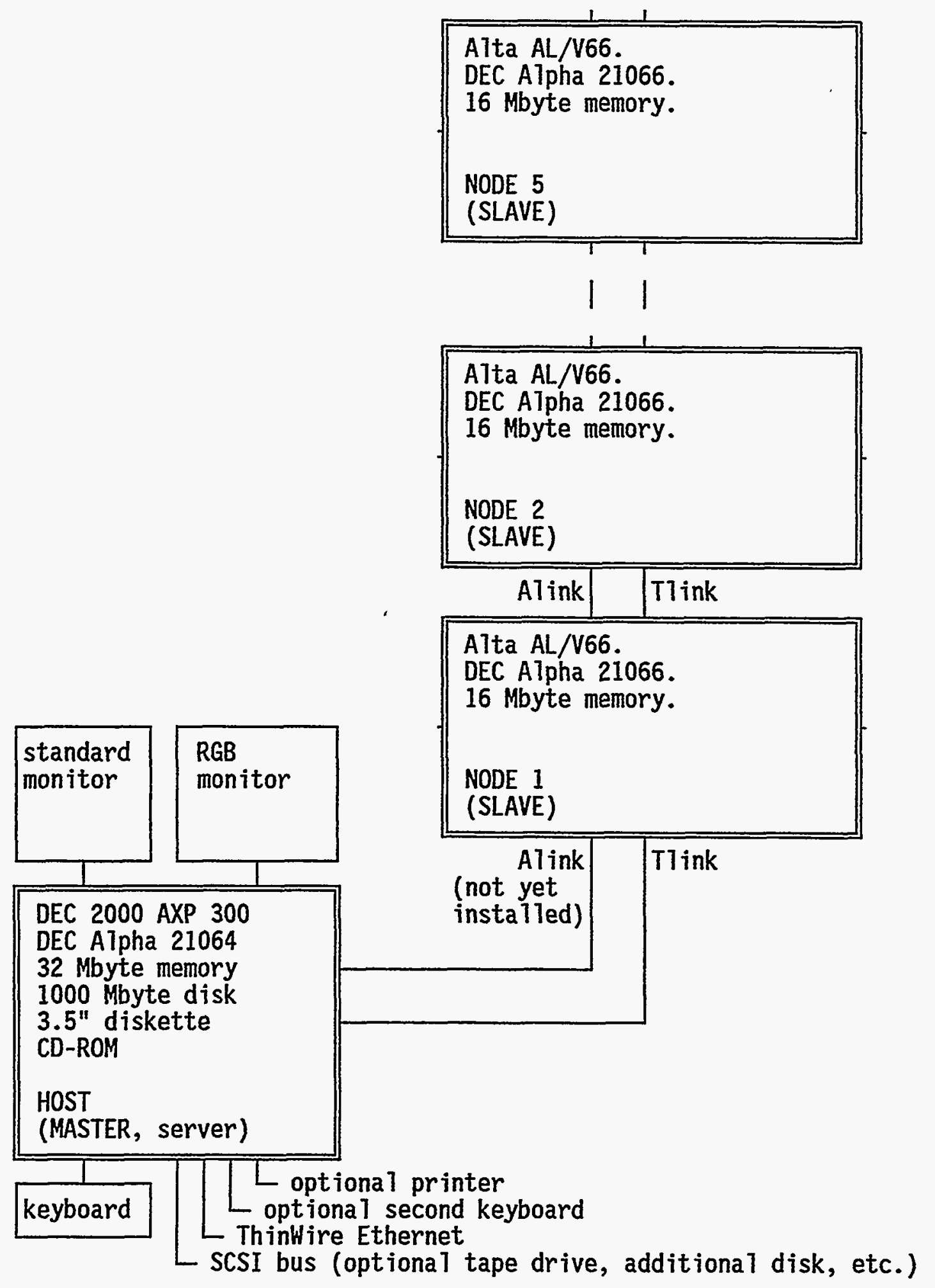

Figure 1: ATR3 nodes. 


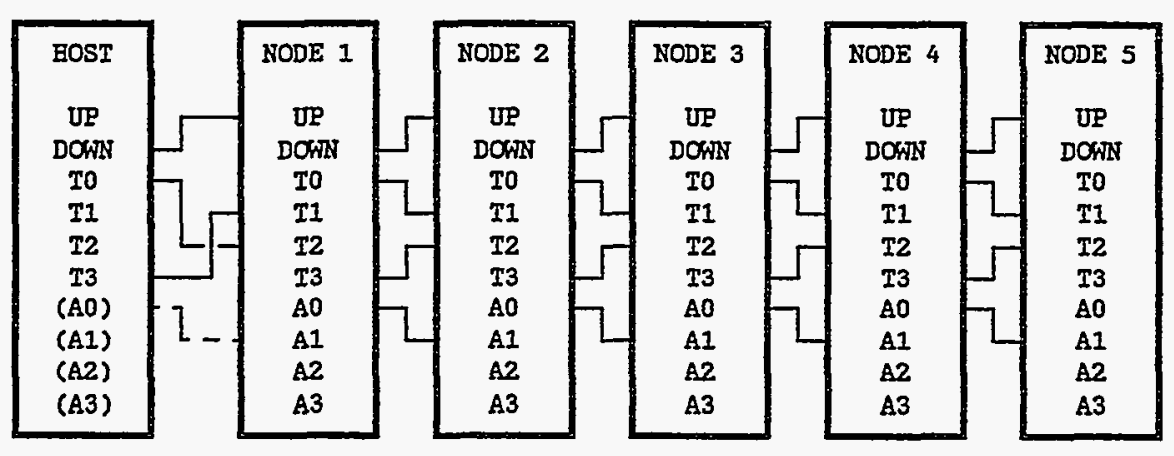

Figure 2: ATR3 inter-node connections. The HOST A-links are not yet installed.

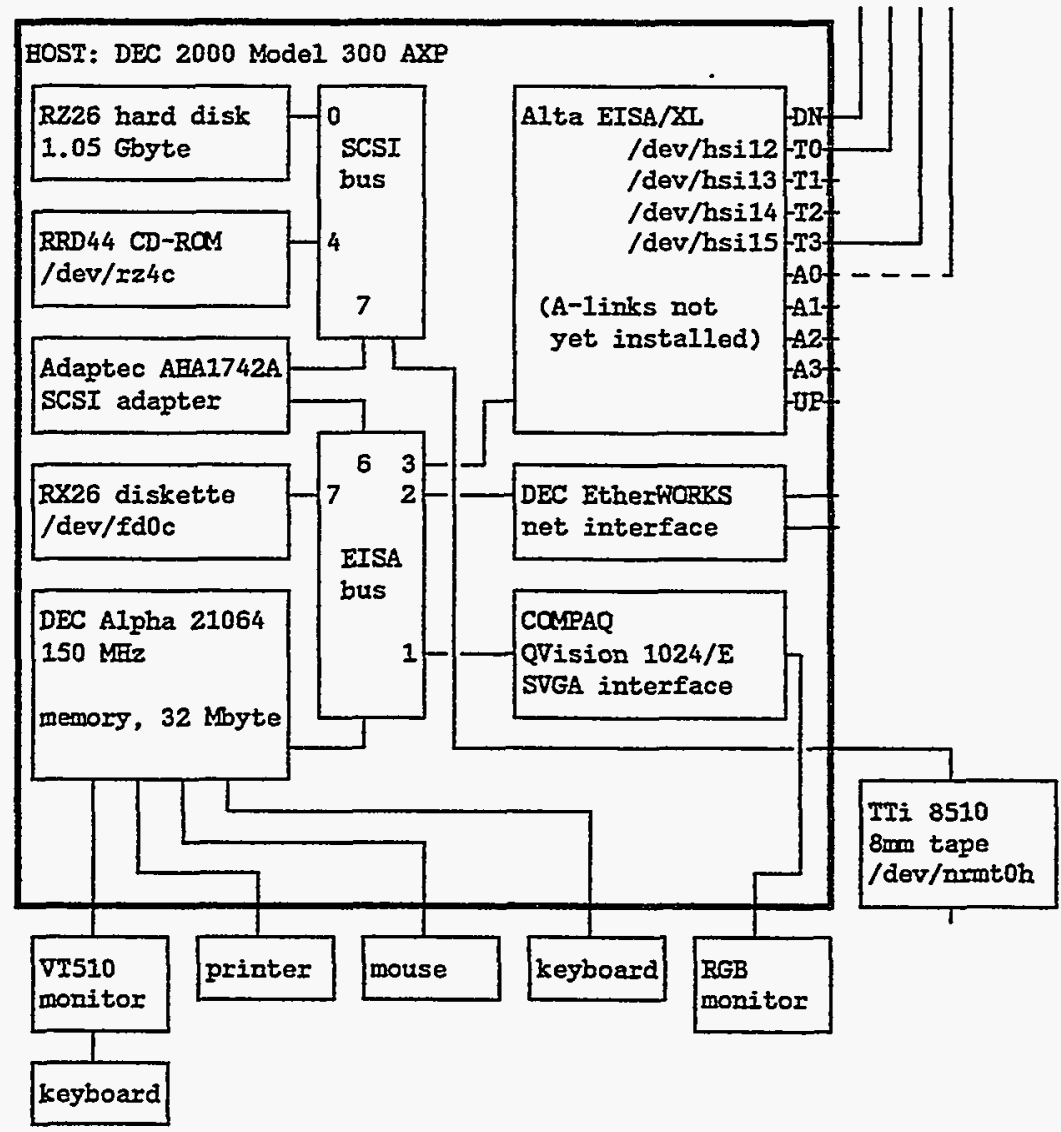

Figure 3: ATR3 host configuration. 


\section{SOFTWARE DESCRIPTION}

The host computer operating system software is OSF/1. The ATR software is written in standard $\mathrm{C}$ augmented with a small library of functions to allow inter-node communication. The software is designed to allow easy addition of new image manipulation functions as the need arises.

There are three programs, called E, F, and G. The three programs have many functions in common, but they also have some differences associated with the different operations they perform. Appendix A lists the functions currently included in the ATR software.

\section{Surveillance Program E}

The surveillance program $\mathrm{E}$ is the production program, used in the field to analyze a set of one or more images of a scene and report on whether there is a target present in the scene. Program E uses a set of three control files: a .sl file, which specifies the names of the files that hold the image data to be analyzed; a .fc file, which includes a sequence of image manipulation instructions that is a recipe for determining whether a target is present in the scene; and, a .cmd file, which contains primarily just the names of the .sl and .fc files. Program E is used very simply, by typing "em" and then, when asked, the name of the .cmd file to be used for this application. Thus, using program $\mathrm{E}$ is trivial after the three control files are ready. Preparation of these files is not trivial; it will be discussed in more detail in a separate report. For the present, we note that the .fc file may use the values of coefficients determined by program $F$.

\section{Training Program F}

The training processes is implemented in program $\mathrm{F}$. The training process is optional. In some applications, the analyst may know by other means how to manipulate the image data to find targets, and he can build this procedure into the program $E$.fc file without ever using program F.

The training process in program $\mathrm{F}$ is used when the target/no target decision is expressed as a linear combination of the raw images or any other images that may be derived from the raw images. Program F gives the optimal values of the coefficients in the linear combination. This is a surprisingly versatile process. Even though the optimized combination is linear, the overall target detection process is not restricted to linear combinations of the raw images, since any of the images in the linear combination can be constructed by any arbitrary non-linear process from the raw images.

In addition to the optimal coefficient values, program $F$ gives a predicted error rate for target detection based on the present training data. Program $\mathrm{F}$ also indicates the importance of each of the images in the linear combination, so that the analyst can simplify the target detection process by eliminating the unimportant parts.

Program F uses training data, in the form of sets of images in which the analyst has marked some areas as targets and other areas as background. This marking of training scenes is done using program $\mathrm{G}$. 


\section{Mask Creation Program G}

For each training scene, a skilled operator must create a mask that indicates which parts of the scene are targets, which parts are background, and which parts are not designated as either target or background regions. Program $G$ is a tool to help the operator do this task. G creates a mask, an "image" that contains the operator's target/background designations, and makes the mask available for program $F$ to use along with the raw training scene images in the optimization process.

Unlike program $E$ and program $F$, program $G$ does not take its instructions from control files; program $G$ takes instructions from the keyboard. Program $G$ includes all of the image manipulation functions that are in program $E$ and program $F$ (but not the coefficient optimization procedure used in the training process), so program $G$ can be used as an interactive version of the software, which some users might find convenient for experimentation when developing a target recognition process.

\section{SYSTEM TESTS}

The three programs E, F, and $G$ have been verified as being functional on the ATR3 hardware. They are generally faster, and is some small ways slightly easier to use, than they are on the other two hardware systems we have tested.

An important aspect of this ATR systems is its speed, primarily the speed of the parallel processor network. We have done direct measurements of speeds for certain typical image analysis operations, and we have done comparisons with two other ATR systems using essentially the same software on different hardware systems (ATR1 and ATR2). ATR1 uses T805 transputers, and ATR2 uses T9000 gamma transputers. The results of the measurements reported here are in some cases different from similar measurements reported earlier ${ }^{1}$, primarily because of improvements in the ATR3 system software and also because of changes in our own software. For these timing measurements, ATR3 was used with only 3 daisy nodes, to make it more directly comparable with ATR2. The times quoted here were measured with the echo turned off, using images with 512 columns by 480 rows of pixels. Some explanation of these times follows:

\section{COMMAND, comments}
ATR1 seconds
ATR2 seconds
ATR3 seconds

This is the format for the following entries. The command, capitalized, and a comment in italics are in the first line; the execution times for each of the three hardware systems, in seconds, are in the second line; discussion is in following lines. The \pm values given with the times are not statistical uncertainties; they are absolute limits on the accuracy of the time measurements, imposed by the limited resolution of the accessible clock.

;remark, remark with leading semicolon

$$
<0.0001<0.0001<0.0001
$$

This is the time required for the master to read a command from the feature calculation file and dispose of it without any attempt at command interpretation. The times listed here are for a remark of minimal length, ";" with no real remark text. These times are strongly affected 
by the time required to read a line of ASCII characters from a disk file; the times will be greater for longer remarks.

REM, remark without echo

$$
0.0003 \pm 0.0001 \quad 0.0001 \pm 0.0001<0.0001
$$

This is the time required for the master to read a command from the feature calculation file and do minimal command interpretation. Command interpretation times are different for different commands and they are generally greater for greater numbers of parameters. The time will of course increase if the echo is turned on. The times listed here are for a remark of minimal length, "REM" with no real remark text.

SET_AB, set scale factors

$$
0.0076 \pm 0.0001 \quad 0.0031 \pm 0.0001 \quad 0.0003 \pm 0.0001
$$

The master sets the values of two variables in a table in the master program, without interacting with the slaves.

\section{CLEAR, clear memory, with no memory de-allocation}
$0.0019 \pm 0.0001$
$0.0008 \pm 0.0001$
$0.0017 \pm 0.0001$

The master writes values to a table in memory and sends a short message to all the slaves (daisy chain nodes), so this example includes minimal one-way master-to-slave communication. Each slave also writes a table in its own memory and, if images or kernels (operators) are defined, frees those memory blocks. No memory de-allocation was done in this test.

READY, wait until slaves are finished, with slaves not busy

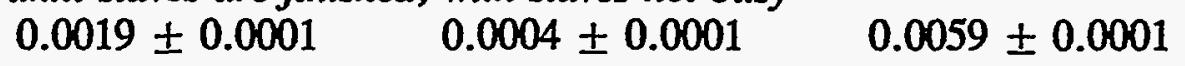

The master sends a short message to all the slaves. Each slave responds separately, and the master waits for all the slaves to respond. Thus, this command involves brief two-way communication between master and slave, with essentially no other operation.

For the three commands SET_AB, CLEAR, and READY, the ATR3 times are in the order expected when inter-node communication time dominates calculation time. For ATR1 and ATR2, the SET_AB command apparently requires more time to do less than the CLEAR and READY commands. This is believed to be because the SET_AB command requires a function call with three parameters, whereas the other two commands use function calls with no parameters. Times being dominated by calculation rather than communication would also account for the CLEAR time being greater than the READY time for ATR2.

DEFKERN, define a kemel (operator)

$$
\begin{array}{lll}
0.0132 \pm 0.0001 & 0.0048 \pm 0.0001 & 0.0100 \pm 0.0001
\end{array}
$$

This operation allocates memory for a local convolution type of kernel (operator), with the whole kernel being stored in each slave's memory. This operation does not set the values of the kernel. The master sends a short message to all the slaves, and writes a few values to a table in memory. Each slave writes a few values to memory, allocates a memory block, and sends a short message back to the master. If the kernel was already defined, the slave also deallocates the old memory block; this de-allocation was done in this test. 
DEFIMG, define an image, with no overlap rows

$$
\begin{array}{lll}
0.0114 \pm 0.0001 & 0.0040 \pm 0.0001 & 0.0098 \pm 0.0001
\end{array}
$$

$D E F M G$, define an image, with 10 overlap rows

$$
0.0115 \pm 0.0001 \quad 0.0040 \pm 0.0001 \quad 0.0098 \pm 0.0001
$$

This operation allocates memory for an image, with different parts of the image being stored in different slave's memories. This operation does not set any pixel values in memory. The master sends a short message to all the slaves and writes a few values to a table in memory. Each slave calculates which part of the image it should store in its own memory, allocates a memory block, and sends a short message to the master. If the image was already defined, as it was in this test, the slave also de-allocates the old memory block. Unlike an earlier version of this software, no significant difference in time is expected when the number of overlap rows is changed. This DEFIMG operation sends slightly shorter inter-node messages than the DEFKERN operation.

ZEROIMAGE, zero an image, with no overlap rows

$$
\begin{array}{lll}
0.0250 \pm 0.0010 & 0.0580 \pm 0.0010 & 0.0045 \pm 0.0005 \\
& \text { calcs }=1.44 & \text { calcs }=18.52
\end{array}
$$

ZEROIMAGE, zero an image, with 10 overlap rows

$A D D$, add two images

$$
\begin{array}{lll}
0.0350 \pm 0.0010 & 0.0640 \pm 0.0010 & 0.0045 \pm 0.0005
\end{array}
$$

$$
\begin{array}{lll}
0.043 \pm 0.001 & 0.124 \pm 0.001 & 0.038 \pm 0.001 \\
& \text { calcs }=1.16 & \text { calcs }=3.77
\end{array}
$$

MUL, multiply two images

$$
\begin{array}{lll}
0.049 \pm 0.001 & 0.117 \pm 0.001 & 0.038 \pm 0.001 \\
& \text { calcs }=1.40 & \text { calcs }=4.30
\end{array}
$$

$D T$, divide two images

$$
\begin{array}{lll}
0.239 \pm 0.001 & 0.351 \pm 0.001 & 0.066 \pm 0.001 \\
& \text { calcs }=2.27 & \text { calcs }=12.07
\end{array}
$$

SQRT, square root of an image

$$
\begin{array}{lll}
0.288 \pm 0.001 & 0.286 \pm 0.001 & 0.080 \pm 0.001 \\
& \text { calcs }=3.36 & \text { calcs }=12.00
\end{array}
$$

$A B S$, absolute value of an image

$$
\begin{array}{lll}
0.105 \pm 0.001 & 0.159 \pm 0.001 & 0.014 \pm 0.001 \\
& \text { calcs }=2.20 & \text { calcs }=25.00
\end{array}
$$

MAXCON, clip low end values of an image, if all pixels are clipped

$$
\begin{array}{lll}
0.055 \pm 0.001 & 0.134 \pm 0.001 & 0.014 \pm 0.001 \\
& \text { calcs }=1.37 & \text { calcs }=13.10
\end{array}
$$

MAXCON, clip low end values of an image, if no pixels are clipped

$$
\begin{array}{lll}
0.052 \pm 0.001 & 0.119 \pm 0.001 & 0.014 \pm 0.001 \\
& \text { calcs }=1.46 & \text { calcs }=12.38
\end{array}
$$

$A D D C O N$, add a constant to an image

$$
\begin{array}{lll}
0.037 \pm 0.001 & 0.096 \pm 0.001 & 0.012 \pm 0.001 \\
& \text { calcs }=1.28 & \text { calcs }=10.28
\end{array}
$$

MULCON, multiply an image by a constant

$$
\begin{array}{lll}
0.042 \pm 0.001 & 0.104 \pm 0.001 & 0.012 \pm 0.001 \\
& \text { calcs }=1.34 & \text { calcs }=11.67
\end{array}
$$


DIVCON, divide an image by a constant

$$
\begin{array}{lll}
0.044 \pm 0.001 & 0.120 \pm 0.001 & 0.012 \pm 0.001 \\
& \text { calcs }=1.22 & \text { calcs }=12.22
\end{array}
$$

For each of these simple arithmetic image manipulation operations, the master sends a short message to all the slaves. Each slave does the indicated pixel-by-pixel operation for its part of the specified images. There is no inter-slave communication. The bulk of the time is for the actual image manipulation. These times should be inversely proportional to the number of slave nodes, except for the ZEROIMAGE operation with overlap rows.

\section{CONVOLVE, correlate an image with a kernel (operator), for a $5 \times 5$ pixel kernel}

$$
\begin{array}{lll}
1.56 \pm 0.01 & 3.09 \pm 0.01 & 0.30 \pm 0.01 \\
& \text { calcs }=1.68 & \text { calcs }=17.33
\end{array}
$$

The master sends a short message to all the slaves. Each slave does the correlation calculation directly (no Fourier transform) for its part of the image. This operation normally must be preceded by the OVERLAP operation to set the values of the pixels in the overlap rows. This time should be inversely proportional to the number of slave nodes and roughly proportional to the size of the kernel domain.

SSQ, local sum of squares, for $5 \times 5$ pixel kernel

$$
\begin{array}{lll}
1.97 \pm 0.01 & 3.43 \pm 0.01 & 0.37 \pm 0.01 \\
& \text { calcs }=1.91 & \text { calcs }=17.75
\end{array}
$$

The master sends a short message to all the slaves. Each slave calculates a weighted sum of squares of values of the pixels in the local region indicated by the domain of the specified kernel, with weights equal to the kernel values. This operation normally must be preceded by the OVERLAP operation to set the values of the pixels in the overlap rows. This time should be inversely proportional to the number of slave nodes and roughly proportional to the size of the kernel domain.

OVERLAP, set pixel values in overlap rows, for 2 overlap rows

$$
\begin{array}{lll}
0.0169 \pm 0.0001 & 0.0076 \pm 0.0001 & 0.0300 \pm 0.0010 \\
& \text { comms }=2.22 & \text { comms }=0.56
\end{array}
$$

OVERLAP, set pixel values in overlap rows, for 10 overlap rows

$$
\begin{array}{lll}
0.0604 \pm 0.0001 & 0.0303 \pm 0.0001 & 0.0800 \pm 0.0010 \\
& \text { comms }=1.99 & \text { comms }=0.755
\end{array}
$$

The master sends a short message to all the slaves. The slaves exchange the values of the pixels in the overlap rows; thus, there is a lot of inter-slave communication, with one long message in each direction for each overlap row. This time is expected to be roughly proportional to the number of overlap rows and independent of the number of slave nodes.

SMTHX, smooth in $X$ direction, for 5 passes

$$
\begin{array}{lll}
0.720 \pm 0.010 & 1.140 \pm 0.010 & 0.105 \pm 0.001 \\
& \text { calcs }=2.11 & \text { calcs }=22.86
\end{array}
$$

The master does a short calculation and sends a short message to all the slaves. Each slave smooths its own part of the indicated image without communication with other slaves. The smoothing function is more Gaussian for more passes, with 5 passes being a good approximation to a real Gaussian and 1 pass having a sharp cusp in the smoothing function. The time is proportional to the number of passes and inversely proportional to the number of slave nodes. 
SMTHY, smooth in $Y$ direction, for 5 passes

$$
1.87 \pm 0.01 \quad 2.08 \pm 0.01 \quad 9.10 \pm 0.10
$$

This is like SMTHX, $\mathrm{X}$ direction smoothing, except that this $\mathrm{Y}$ direction smoothing requires significant inter-slave communication. Two short messages per image column per pass are sent by each slave. This time should be roughly inversely proportional to the number of slave nodes only if the calculation time dominates the communication time. The disproportionately long time required by ATR3 for this operation is believed to be due to communication overhead.

\section{MEDIAN, two-dimensional median filter, for a 21-pixel window}

$$
\begin{array}{lll}
11.7 \pm 0.1 & 17.9 \pm 0.1 & 1.8 \pm 0.1 \\
& \text { calcs }=2.18 & \text { calcs }=21.67
\end{array}
$$

The master sends a short message to all the slaves. Each slave does the calculation for its part of the image without inter-slave communication. This operation normally must be preceded by the OVERLAP operation to set the values of the pixels in the overlap rows. This time should be inversely proportional to the number of slave nodes.

UNDERSAMPLE, decimate (undersample) an image, for decimation by a factor of 2 in each direction with a pre-defined destination image

$$
\begin{array}{lll}
0.019 \pm 0.001 & 0.114 \pm 0.001 & 0.010 \pm 0.001 \\
& \text { calcs }=0.555 & \text { calcs }=6.33
\end{array}
$$

The master sends a short message to all the slaves. If the destination image is not already defined, this operation defines it; in this test, the destination image was already defined. This time should be inversely proportional to the number of slave nodes. For unknown reasons, ATR2 seems to have an anomalously low speed for this operation.

SCALE, scale an image

$$
0.175 \pm 0.001 \quad 0.354 \pm 0.001 \quad 0.037 \pm 0.001
$$

The master sends a short message to all the slaves. Each slave scans its part of the specified image for the maximum value, and then the slaves daisy-chain communicate the maximum value to the master. The master then sends a short message to all the slaves, and each slave multiplies its part of the image by a constant. Thus, there is some master-slave and interslave communication involved in this scaling operation. This time should be approximately inversely proportional to the number of slave nodes.

READIMAGE, read an image

$$
\begin{array}{lll}
1.50 \pm 0.10 & 1.10 \pm 0.10 & 1.00 \pm 0.10 \\
& \text { comms }=1.36 & \text { comms }=1.5
\end{array}
$$

WRITEIMAGE, write an image

$$
\begin{array}{lll}
2.40 \pm 0.10 & 1.85 \pm 0.05 & 1.25 \pm 0.05 \\
& \text { comms }=1.30 & \text { comms }=1.92
\end{array}
$$

These times are for the host system disk. Accessing the ATR1 SCSI disk takes a little longer. These times should be essentially independent of the number of slave nodes. They depend on external factors such as the disk speed, the bus speed, and perhaps the host operating system or other data transfer software. 


$\begin{array}{lll}Q U A D U V, \text { fit every local region with a quadratic polynomial, for a } 21 \text {-pixel kernel } \\ 108.0 \pm 1.0 & 147.0 \pm 1.0 & 41.0 \pm 1.0 \\ & \text { calcs }=2.45 & \text { calcs }=8.78\end{array}$

This requires a substantial amount of calculation, including a 6-parameter least squares fit, for every pixel. This time should be inversely proportional to the number of slave nodes.

$B R A N C H$, in a minimal loop

$\begin{array}{rrr}0.0218 \pm 0.0002 & 0.0086 \pm 0.0001 & 0.0012 \pm 0.0001 \\ \text { JUMP, in a minimal loop } & & \\ 0.0222 \pm 0.0002 & 0.0088 \pm 0.0001 & 0.0013 \pm 0.0001\end{array}$

The times for BRANCH and JUMP commands vary greatly, depending mainly on the length of the feature command file, particularly that part of the file before the command. The times will also depend on disk file access times or disk caching capabilities. These times are for unrealistically small feature command files. These times depend strongly on the speed of the host computer for command files of significant size.

In systems of this type, two different speeds are of interest: the speed of calculations within a node, and the speed of communication between nodes. Which is more important depends almost entirely on the particular application. Our experience suggests that calculation speed is generally more important in automatic target recognition. In the above timing measurements, some times are dominated by calculation time, and some by communication time.

For those timing measurements that are almost totally indicative of calculation time, a value is given for "calcs" for ATR2 and ATR3. This is the relative calculation speed per node, relative to ATR1. It is calculated by

$$
\text { calcs }=\frac{(\text { ATRI time }) *(\text { number of ATRI nodes })}{(\text { ATR2 time }) * \text { (number of ATR2 nodes })}
$$

for ATR2, with the obvious analog for ATR3. The values for this speed ratio vary significantly for different types of operations, because of the internal differences in the processors. There is a tendency toward a greater speed advantage for ATR2 and ATR3 for the more complicated calculations. We use as the representative calculation-intensive operation the two-dimensional median filter, MEDIAN. For this operation, ATR2 has a relative speed per node of 2.18, and ATR3 has a relative speed per node of 21.67. The important relative calculation speed per cost, which is simply the relative speed per node divided by the relative cost per node, is about 1.17 for ATR2 and 2.32 for ATR3.

This software package contains no operations that are purely communication. Even such operations as reading an image from a disk file involve a significant amount of calculation, in calculating array indexes and converting from 8-bit integers to 32-bit floating point values. Perhaps the best indication we have of relative communication speed is the OVERLAP operation, in which 512 floating point values (2048 bytes) per data transfer are exchanged between neighboring processors without type conversion and the only arithmetic is array index calculation. For this, like most inter-node communications, it is not reasonable to speak of speed per node, because the time of the overall operation usually does not depend significantly on the number of nodes. In the typical inter-node communication, there is in effect a parallelism with many pairs 
of nodes communicating simultaneously, so that the overall time is essentially the time required for any one pair of nodes to communicate. Thus, we represent the relative communication speed by the ratio of the times:

$$
\text { comms }=\frac{(\text { ATR1 time })}{\text { (ATR2 or ATR3 time) }} .
$$

This relative communication speed, as measured for the OVERLAP operation for 10 overlap rows, is 1.99 for ATR2 and 0.755 for ATR3. The communication slowness of ATR3 is surprising, in light of the raw A-link speed of $250 \mathrm{Mbit} / \mathrm{second}$ compared with the ATR1 T-link speed of $20 \mathrm{Mbit} / \mathrm{second}$. The ATR3 system has a substantial per-message system software overhead for inter-node communication. This is apparent in the very long time required for ATR3 to do the SMTHY operation, which involves many short messages exchanged between neighboring nodes. The ATR3 communication speed is much better for longer messages.

The final version of the parallel processor system software was not yet available when these timing measurements were done. We know that the final system software will allow streamlining certain parts of the ATR software, and we speculate that this will significantly increase the inter-node communication speed. No measurable change is expected in calculation speed.

\section{CONCLUSIONS}

The ATR3 hardware is complete except for the expected addition of A-links to the host. This addition will not adversely affect the system operation in any way; it may enhance the communication speed, but probably not significantly for the present ATR software. The existing hardware is fully operational, with no known defects.

We expect that the parallel processor system software will be enhanced by the addition of certain inter-node communication functions, which might significantly increase the communication speed of this ATR3 system.

The existing ATR3 system is fully operational. It is a computationally powerful system for target recognition and other image manipulation applications. The software is easily expandable; new functions can be incorporated into the existing framework with minimal difficulty. The hardware is also expandable, in that additional parallel processor nodes can be added to increase the speed and total memory of the system.

\section{REFERENCE}

1. J. K. Partin, G. D. Lassahn, and J. R. Davidson, Automatic TLI Recognition System Hardware Implementations, INEL-95/0501, October 1995. 
APPENDiX A

\section{E14, F14, and G14 COMMANDS}




\section{E14, F14, and G14 COMMANDS}

\section{CONTROL OF PROGRAM}

REM, ECHO ON, ECHO OFF, ECHO, ECHOTIME, READY

LABEL, JUMP, BRANCH, IF, PAUSE, STOP, END

GOSUB, SUBDEF, RETURN

\section{DEFINING IMAGES AND KERNELS (OPERATORS)}

DEFIMG, DEFKERN, COPDEF, CLEAR, SET_AB

also READIMAGE, READSCENE, RESAMPLE, UNDERSAMPLE, NEWIMG

READING IMAGES AND RERNELS FROM FILES

READIMAGE, READSCENE, READKERNEL, BANDR, PHEAD; also INCFIL

INM, INS

BASIC IMAGE OPERATIONS

ZEROIMAGE, SETPIX, NTRP00, NTRP01, COPY, COPYEDGE, OVERLAP, RESAMPLE, UNDERSAMPLE, INSERT, EXTRACT, REMAP, REGISTER

\section{ARITHMETIC OPERATIONS ON IMAGES}

MAX, MIN, MAXCON, MINCON, REPLO, REPHI, ADD, SUB, MUL, DIV, ADDCON, SUBCON, MULCON, DIVCON, SQRT, ABS, TAN, ATAN, ATAN2, LOG, EXP: All the images in these operations must be already defined, all the same size, and all distributed the same way among the daisies. The images need not be different; dst can be the same as src or src2, etc. const is a single floating point value. These operations do not use or set the values of overlap rows.

CONVOLUTION and related operations

CONVOLVE, SSQ

GRADIENTS

GRADT, XGRAD, YGRAD, GRADCON; also XY2RT

FILTERING IMAGES

SMTHX, SMTHY, MEDIAN, MED1X, MED1Y, SMTHXS, SMTHYS

MISCELLANEOUS IMAGE OPERATIONS

LIN01, LIN02, XLIN01, NLIN01, EDJ02

MODMSK

QUADXY, QUADUV

SEGLAB

RIJCON, GRADCON

MOMUV

PEAK1, PLNK1, PMRG1, PACC1, PMOMXY1, PMOMUV1, PMOMXY, PMOMUV

XIMG, YIMG 
GEOMETRIC CORRECTIONS

SBEND

DISPLAYING IMAGES

VGA, DISP, DISPRES; also SCALE, CONTOUR

IMAGE INFORMATION

STATS, SUMPIX, HIST2, PDFXYZ, PDFXY1; SET_AB, DEFIMG

FEATURE IMAGES

WRITEFEAT, FEATFIL, FEAT*; also SCALE

WRITING IMAGES TO FILES

WRITEIMAGE, WRITEFEAT, WRITERESULT; also INCFI, SCALE OUTM, OUTS

\section{SCALAR VARIABLES}

PRINTS, SABS, SADD, SDEFF32, SDEFI32, SDIV, SEQ, SMAX, SMIN, SMUL, SSQRT, SSUB (formerly called PRINTU, UABS, UADD, UDEFF32, UDEFI32, UDIV, UEQ, UMAX, UMIN, UMUL, USQRT, USUB), STAN, SATAN, SATAN2, SLOG, SEXP

The user can define variables, either 32-bit integers or 32-bit floating, set their values, print their values, and use them as parameters in commands. Some scalar variables are predefined and their values are automatically set by certain operations. The pre-defined scalar variables are named \$N, \$NCOL, \$NROW, \$SCENE, \$MINI, \$MAXI, \$MINJ, \$MAXJ (integers), and \$AVG, \$SIG, \$MAX, \$MIN, \$A, \$B, \$AVGX, \$AVGY, \$SIGX, \$SIGY, and $\$ C O X Y$ (floating). The program DOES distinguish between upper and lower case characters in variable names. The arithmetic operations for scalar variables allow free mixing of integer and floating types.

\section{STRING (FILE NAME) MANIPULATION} SEQ, SADD, INCFIL, PRINTS

The user can manipulate certain file names that are stored in the program. These are \$FSIA[n], an array of scene image file names; \$FFIA[n], an array of feature image file names; and \$FRI, the result image file name. The user cannot define his own file name or string variables.

The command names themselves are NOT case sensitive; the command names can be typed in upper case, lower case, or any mixture of cases. The user-accessible and user-defined variable names ARE case-sensitive. Command parameters may be separated by commas or spaces or both. 
ABS dst, src

Set image dst $=$ absolute value of image src.

ADD dst, src1, src2

Set image dst $=$ image src1 + image src2.

ADDCON dst, src, const

Set image dst $=$ image src + const.

ATAN dst, src

Set each image dst pixel equal to the inverse tangent of the value of the corresponding image src pixel. Angles will be in radians, in the interval $(-\pi / 2, \pi / 2)$.

ATAN2 dst, srcy, srcx

The common inverse tangent function with two arguments, corresponding to the opposite and adjacent sides of a right triangle. Set each image dst pixel equal to the inverse tangent value (the angle), with the opposite side value from image srcy and the adjacent side value from image srcx. Angles will be in radians, in the interval $(-\pi, \pi]$.

BANDR nbands

BANDR band, img [, byt0 [, ncol [, bpp ]]]

BANDR (no parameters)

This BANDR command is used to prepare for reading row-interleaved images from a file with the READIMAGE -1 command.

In its first form, with only one parameter, the BANDR command sets the number of bands to nbands.

In its second form, with more than one parameter, BANDR sets parameters for band (or sub-image) number band (band $=1,2, \ldots$ nbands), as follows. img is the number of the memory image to receive band band, or if the img value is zero, band band is not used. byto is the number of bytes from the beginning of the image file composite row to the beginning of band band. ncol is the number of columns (pixels, not necessarily bytes) to be read for band band. bpp is the number of bytes per pixel (see the READIMAGE command) for band band. It is acceptable to skip bands of the file; however, all bands that are specified by this BANDR command should be in order of increasing byt0, the order in which they occur in the file. If the value -1 is given for byt 0 or ncol, or if the value 0 is given for bpp, for any band, the READIMAGE -1 command will attempt to determine the correct values for these parameters from the file header. If values are not specified for the last 1,2 , or 3 parameters in this BANDR command, their values stored in the program are not changed. Initial values for all these parameters are 0 .

With no parameters, the BANDR command prints the current values for all bands for which non-zero parameters have been specified.

\section{BRANCH $\mathbf{n}$}

Jump to the .fc file line which is $n$ lines removed from the line containing this BRANCH statement. $\mathbf{n}$ may be positive or negative. $\mathbf{n}=0$ is an error condition, since this would establish an infinite loop of repeatedly branching to the current line. Although $n=1$ is not an error, a BRANCH command with $\mathbf{n}=1$ is superfluous. It causes a jump to the following line, which 
would be the normal sequence even if the BRANCH statement were absent. (BRANCH does not exist in program G.)

CLEAR (no parameters)

Clear the tables that hold information about images, operators, and user-defined scalar variables, and free the memory allocated for these things.

CONTOUR dst, src, sval, dval

This operation draws one contour curve in destination image dst, representing a curve of constant intensity in source image src. If a pixel in src has intensity at least as great as the floating point value sval, and at least one of its four nearest neighbors has intensity less than sval, then the corresponding pixel in dst is set equal to the floating point value dval. Otherwise, dst pixel values are left unchanged. src should have at least 1 overlap row. dst does not need to be the same size as src.

\section{CONVOLVE dst, src, opr}

Set image dst equal to the convolution of image src with operator (kernel) opr. That is, set each dst pixel equal to the sum of $\left\{\right.$ src*opr $^{*}$, summed over the domain of the operator opr with the operator's "center" pixel positioned on the src pixel that corresponds to the dst pixel. The pixels of image dst in the excluded edge region, which is defined by the domain of the operator opr, are set to zero. src should have enough overlap rows to accommodate operator opr; the overlap rows must be present and have valid values. Correct values can be put into the overlap rows with the OVERLAP command. The number of overlap rows should be at least as large as the larger of the absolute value of $j \max$ or the absolute value of $j \min$ for operator opr. opr must be greater than 0 . dst and src should be different images. The image dst does not need to be the same size as the image src, but the two images do need to bxe distributed among the daisies in a manner compatible with the RESAMPLE algorithms. If there is question about compatibility, it may be helpful to have extra overlap rows for image src.

Note that this is not strictly a proper convolution calculation, which would require reversing the signs of the two indexes in the operator.

\section{COPDEF dst, src [, novl ]}

Define space for previously undefined image dst so that it is compatible with the previously defined image src. That is, the total sizes are the same for the two images, and their primary rows are distributed the same way among the several daisies, so that the two images are compatible for 2- or 3-image operations. The new image dst will have novl overlap rows regardless of how many overlap rows the old image src had. If a value is not given for novl, image dst will be created with the same number of overlap rows as image src. COPDEF does not set any pixel values. COPDEF sets $\$ A$ and $\$ B$ to 0.0 and 1.0 for dst.

COPY dst, src size.

Copy image src to image dst. Both images must be already defined and of the same

COPYEDGES dst, src, opr

Copy the values of the pixels at the edges of image src to the corresponding pixels of image dst. The edge pixels are specified by the domain of the operator opr. That is, if a 
certain pixel of image src can be used as the center pixel of the operator opr without the domain of the operator opr extending off the image src, then that certain pixel is NOT an edge pixel and it will not be copied. This same definition of edge regions in terms of an operator is used in many of the operations described later in this report. opr should be greater than 0 for this operation, since it would not make sense to copy an edge region which contains no pixels. Images dst and src should be the same size.

\section{DEFIMG img, ncol, nrow [, novl] \\ DEFIMG img \\ DEFIMG (no parameters)}

The first form of DEFIMG, with 4 parameters, reserves memory in each daisy to hold part of image img. novl is the number of overlap rows. If no value is given for novl, the value 0 is used. The full image comprises ncol columns by nrow rows. If either ncol or nrow is 0 , the image img is un-defined; its memory space is deallocated and any image data is lost.

You do not have control over which part of the image is held by which daisy. Two images of the same size may be distributed differently among the several daisies, if the two images do not have the same number of overlap rows or if they were created by different processes; such image pairs are not compatible for most multiple-image operations. If two (or more) images are defined by this DEFIMG command with the same values of all 3 parameters ncol, nrow, and novl, they will be compatible for two-image operations. See the COPDEF command.

DEFIMG does not set pixel values. DEFIMG sets the scale factors A and B for image img to 0.0 and 1.0 .

The second form of DEFIMG, with only one parameter, sets the user-accessible variables \$NCOL, \$NROW, and \$N to the number of columns, number of rows, and number of overlap rows in image img. If the echo is on, these values are printed on the screen.

The third form of DEFIMG, with no parameters, lists on the screen the image number, number of rows, number of columns, and number of overlap rows for all defined images.

DEFKERN opr, imin, imax, jmin, jmax

DEFKERN opr

DEFKERN (no parameters)

The first form of DEFKERN, with 5 parameters, reserves memory in each daisy to hold all of the convolution operator (or kernel) opr. The parameters imin, imax, jmin, jmax define the domain of this operator, in the horizontal (positive to the right) and the vertical (positive downward) directions respectively, relative to the "center" pixel. The "center" pixel does not need to be in the center, or even in the two-dimensional domain specified by imin, imax, jmin, jmax. The "center" pixel is merely the pixel with operator domain coordinates $i=0$ and $j=0$, which is often, but not necessarily, in the center of the domain. opr must be greater than 0 . Operator 0 is always automatically defined with imin, imax, $\mathrm{jmin}, \mathrm{jmax}$ all equal to zero; this is useful as a dummy operator with a one-pixel domain. Using operator 0 to specify an excluded edge region implies that none of the image is excluded.

The second form of DEFKERN, with only one parameter, sets the user-accessible variables \$MINI, \$MAXI, \$MINJ, and \$MAXJ equal to the corresponding values for operator opr. If the echo is on, these values are printed on the screen.

The third form of DEFKERN, with no parameters, lists on the screen all the defined kernels and their domain limits. 


\section{DISP caption, img [, row0 [, col0 [, Q ]]]}

Display image img on the high-resolution RGB monitor. The top left corner of the image will appear at row0,col0 in the screen display. Red will be used for pixels with value less than $\mathbf{Q}$, green for pixels with value greater than $\mathbf{Q}$. If a value is not given for $\mathbf{Q}$, the value 0.0 is used. If values are not given for row 0 and colo, values 0 and 0 are used. Pixel values should be between 0.0 and 255.0 (see SCALE). The character string caption will be printed below the image on the screen. caption should not contain any embedded spaces. If a "." is the first character of the string given as the caption, then the last-used file name will be used as the caption. If the echo is on, the image will remain on the screen, and the program will stop execution, until the operator hits a key on the keyboard. The ATR2 system uses the environment variable QT9. ATR3 uses the environment variable DISPLAY, and assumes that the window manager program mwm is running in the background.

\section{DISPRES caption, img, row0, col0, $Q$, res}

This is like DISP, except that the pixels are colored red or green according to whether the corresponding pixel in the image res (normally, but not necessarily, a result image) has a value less than $\mathbf{Q}$. This allows an input image to be displayed with color coding based on a result image.

DIV dst, src1, src2

Set image dst $=$ image src1 / image src2.

DIVCON dst, src, const

Set image dst $=$ image src $/$ const.

ECHO (no parameters except a remark)

Echo this input line back to the screen if the echo is on.

\section{ECHO ON (no parameters)}

Turn on the echo, so that commands are printed on the operator's screen as they are executed, along with certain related information including warnings of possible errors. The default condition is echo on.

ECHO_OFF (no parameters)

Turn off the echo.

ECHOTIME (no parameters except a remark)

Echo the time and this input line to the screen, regardless of the setting of the echo parameter. This is useful for timing studies and diagnostics.

\section{EDJ02 bright, tangle, src, skr, opr, length, width, avg, g}

Find edges. This EDJ02 uses XGRAD followed by LIN02 and YGRAD followed by LIN02 to find sharp gradients in the image src. Image bright is set to the magnitude of the gradient, and image tangle is set to the tangent of the angle between the gradient direction and the $\mathrm{x}$-axis. The opr, length, width, and avg parameters are used in LIN02. $g$ is a gradient threshold; gradient amplitudes less than $\mathrm{g}$ are set to zero and ignored. skr is the number of an image than can be used for scratch space. It does not matter whether image skr is previously 
defined; this operation will define, use, and destroy image skr. This EDJ02 operation requires one overlap row for image src. All four images should be different, all of the same size. bright pixels in the excluded edge region defined by the domain of opr are set to zero.

\section{END (no parameters)}

Indicate the end of the feature calculation script in the .fc file, and also stop the feature calculation process for the current scene. The END differs from the STOP command in that a BRANCH or JUMP or GOSUB command can cause the program to skip over a STOP command and execute later commands, but the program will never skip over an END command and will never read any of the .fc file after the first END command. Each script in a .fc file should contain at least one STOP or END command, and it might contain more than one STOP command. END should not be used as part of an IF command.

\section{EXP dst, src}

Set each image dst pixel equal to the exponential (inverse natural logarithm) of the corresponding image src pixel.

\section{EXTRACT dst, sTc, row, col}

Copy all of image dst from the region of image src with top left corner at row row, column col. If the requested region does not lie entirely within image src, part of the destination image dst is left unchanged. dst and src should be different images. This operation does set the overlap rows in dst.

\section{FEATFIL [-1]}

FOR PROGRAM F ONLY. Read a file name from the scene list file and enter that file name into the internal list of feature image file names, without actually reading or writing any image file. The " + " and "." file name constructs, used in the READSCENE command, also apply here. This command is useful in the F program when the feature images are the same size as the scene images, in which case the program can use the same file for both feature and scene masks and does not need to write a separate file for the feature mask. Note that the feature mask file name should always be the first name in the list of feature image names.

If the -1 parameter is given, the file name used by FEATFIL is not stored as the last-used scene file name. Thus, the next call to READSCENE or FEATFIL with a reference to the previous scene image file name (" + " or ".") does not use the file name referenced in this call to FEATFIL but uses the previously stored scene image file name.

\section{FEAT* sTc1, sTc2}

FOR PROGRAM F ONLY. The program F maintains an internal list of feature images that have been defined, and this list is used during the calculation of the sums in F. Feature images are defined by a WRITEFEAT, a FEATFIL, or a FEAT* command. They are automatically assigned sequence numbers as they are defined. The first feature image, number 0 , must always be the mask. The first "real" feature image is number 1 . This operation FEAT* puts a special code into the list of defined feature images. The code directs the sums calculation subroutine to use an additional feature image that is not actually stored in a file; this additional feature image is the product (pixel-by-pixel multiplication) of the two previously defined feature images numbered src1 and src2. (src1 and src2 may be the same.) This allows the use of a feature image that is the product of two other feature images, without taking the time to write 
and read a file for this image. This FEAT* command should not be used until both of the feature images src1 and src2 are defined.

\section{GOSUB subname $[, \ldots]$}

Execute the "subroutine" named subname that is in the .fc file. See the SUBDEF command. The GOSUB command must have as its first parameter the name of the subroutine to be executed. It should usually have additional parameters to match the parameters specified in the subroutine definition, in the SUBDEF command. This software does not check for matching parameter number or type. If the GOSUB command specifies more parameters than the SUBDEF command, the extra parameters are ignored. Depending on the nature of the subroutine, it may be acceptable for the GOSUB command to have fewer parameters than the SUBDEF command specifies.

If a subroutine parameter is a scalar (not an array), the value of the scalar in the GOSUB command is copied to the corresponding variable in the SUBDEF command. If the subroutine changes the value of its variable, that change is NOT made in the corresponding GOSUB parameter value (unless the GOSUB parameter and the SUBDEF parameter are the same variable). If a subroutine parameter is a whole array (not just one element of an array), the address of the GOSUB parameter array is copied to the corresponding SUBDEF parameter, so that both array variables are references to the same array of values in memory. Thus, if the subroutine changes the value of an element of an array parameter, that change is actually made in the GOSUB array element. To specify a whole array as a GOSUB parameter, give the name of the array with no index in brackets. That is, the parameter XXX[3] specifies one element (element number 3, the fourth element) of the array XXX, but the parameter XXX specifies the whole array XXX.

GRADCON dstb, dsta, stcb, stca

Concentrate (or sharpen) the peaks in a vector field, in the direction of the vector. This is intended to concentrate a gradient vector field, such as is obtained from operation GRADT or from the combination of XGRAD, YGRAD, and XY2RT. Image srcb contains the source vector magnitude, image srca contains the tangent of the angle between the vector and the $x$ axis, and images dstb and dsta will contain the corresponding quantities for the concentrated vector field. GRADCON assumes that the single rows and columns of pixels at the edges of the source images are all zero, and it sets these edge pixels to zero in dstb. This operation uses one overlap row for each of the source images. All the images should be of the same size. The destination images need not be distinct from the source images or from each other. (It does not make sense for the two source images to be the same.) If the two destination images dstb and dsta are the same, the destination image will contain the concentrated vector magnitudes. This operation can be repeated to obtain more sharpening, but the OVERLAP operation should be done before each GRADCON operation. This operation is not perfect, but is quite good. There is sometimes some concentration in the wrong direction, which gets worse with repeated application of GRADCON.

\section{GRADT dstr, dstt, sTc}

Set image dstr equal to the magnitude of the gradient, and image dstt equal to the tangent of the angle between the gradient angle and the $\mathrm{x}$ (horizontal) axis, for the gradient of the intensity in image src. This operation uses one overlap row for src. The destination images need not be different. If the two destination images dstr and dstt are the same, the destination 
image will contain the gradient magnitudes and the gradient direction values will not be written to any image. The top and bottom rows, and the left and right columns, of dstr are set to zero. Both dstr and dstt should be different from src, and all three images should be of the same size.

HIST2 src, xcl, nbin, vmin, vmax

Calculate the histogram of intensity values for image src. Operator xcl defines an excluded edge region; $x c 1$ may be 0 . The histogram will have nbin bins representing the pixel values from vmin to vmax, plus two more bins, for valus less than vmin (in bin 0 ) and for values greater than or equal to vmax (in bin nbin +1 ). This operation sets the user-accessible variables $\$ N=$ number of pixels in the histogram range, $\$ A V G=$ average value of those pixels, and \$SIG = standard deviation of those pixel values. These statistics are calculated from the histogram, not directly from the image.

\section{IF $\mathbf{v 1 , ~ o p , ~} \mathbf{2}$, command}

Evaluate the logical statement $\mathbf{v} 1$ op $\mathbf{v} 2$ and, if the statement is true, execute command. $v 1$ and $\mathbf{2} 2$ are scalars, either constants or user-defined scalar variables. op is any of the 6 relational operators " $==$ " (equals), " $=$ " (does not equal), " $<$ " (is less than), " $>$ " (is greater than), "<=" (is less than or equal to), and " $>=$ " (is greater than or equal to). command may be any of the valid commands listed in this appendix, except END. The entire IF command, including command, must be on a single line in the .fc file.

\section{INCFLL filename [, nleft [, nright ]]}

This command increments the file name filename. Only those characters in the base part (not the path or the extension) are incremented. Only the characters from the nleft'th through the nright'th before the extension (or before the end of the file name) are incremented. If nleft or nright is not supplied, the default values allow all the characters in the file name base to be incremented.

"Incrementing a file name" means that we treat the base file name, exclusive of the path and extension, as if it were a kind of string of digits representing a number, and we increase it by one. For example, if we do not give values for nleft or nright, C:FILE15.IMG increments to C:FILE16.IMG; NAME29 to NAME30; FILE9 to FILF0; TESTA to TESTB; F7Z to F8A; XZZ to YAA; TT99 to TU00; etc. The path and extension are never changed. Alphabetic characters always increment to other alphabetic characters, and numerals to other numerals. For nleft $=2$ and nright $=2$, TT99 increments to TT09; for nleft $=3$ and nright $=2$, TT99 increments to TU09.

INM filename [, header [, ncols [, nrow [, row0 [, col0 [, bpp ]IIII]

FOR PROGRAM G ONLY. This command first invokes the READIMAGE operation for image 2, which is the mask image in the mask-generating process. If image 1 is not a compatible scene image, this command defines image 1 to be compatible with image 2 and sets all of its pixels to 0 . Finally, this command invokes the MARK command.

INS filename [, header [, ncols [, nrow [, row0 [, col0 [, bpp ]III]] FOR PROGRAM G ONLY. This command first invokes the READIMAGE operation for image 1 , which is the scene image in the mask-generating process. If image 2 is not a compatible mask image, this command defines image 2 to be compatible with image 1 and sets all of its pixels to 0 . Finally, this command invokes the MARK command. 
INSERT dst, row, col, src

This command copies image src into image dst, with the top left pixel of image src going into dst pixel (row, col). This operation copies only that part of src that will fit into the specified area of dst. src and dst should be different images. This operation does set the overlap rows in dst.

\section{JUMP label}

Jump to the line numbered label in the .fc file. label is normally the parameter in a LABEL statement somewhere in the .fc file, in which case this JUMP command causes a jump to the line containing that LABEL command. label can also be any constant or user-defined variable whose value is a line number. (JUMP does not exist in program G.)

\section{LABEL label}

Set the value of the user-defined variable label equal to the line number of the line containing this LABEL command. If label is not already defined, this LABEL command will define it as a type 132 variable. The intent is that the variable label will be used with the JUMP command and for no other purpose, but label is actually simply another user-defined type 132 variable and it can be manipulated just as any other user-defined variable. Of course, if the value of label is changed after it is set by the LABEL command, later JUMP label commands may produce undesired results. (LABEL does not exist in program G.)

\section{LIN01 bright, tangle, src, opr, length, width}

Find lines in image src. Set each pixel in image bright equal to the brightness of the line (if any) passing through the corresponding pixel in image src, and set the corresponding pixel in image tangle equal to the tangent of the angle between the line and the $x$ (horizontal) axis. LIN01 will not find lines with negative brightness. bright, tangle, and src should all be the same size, and bright and tangle should be different from src. If bright and tangle are the same image, the image will be set equal to the brightness values and the angle information will not be stored in any image. Operator opr contains weights for the local region which is analyzed for the presence of a line. length is the minimum acceptable line length parameter, and width is the maximum acceptable line width parameter. length and width are measured in pixels, but they are floating point values and fractional parts are meaningful. This operation uses overlap rows for image src; it does NOT set any overlap row values in either of the destination images bright or tangle. bright pixels in the excluded edge region defined by the domain of opr are set to zero.

The LIN01 algorithm is designed to find bright lines on a zero-intensity background, with no negative pixel values. One procedure that is usually reasonable is to subtract a smoothed version of the source image from the raw source image, and then use the MAXCON operation to remove negative pixel values, before using LIN01. Dark lines can, of course, be found by negating an image before starting so that the dark lines appear bright. This algorithm treats the src*opr intensity versus position data as a bivariate probability density function, finds the principal axes, and compares the standard deviations in the principal directions with length/sqrt(12) and width/sqrt(12) to determine whether the distribution is "long" and "narrow" enough to be construed as a line. (For a line of uniform intensity, the standard deviations of the distribution are equal to length/sqrt(12) and width/sqrt(12).) In other words, this algorithm looks at the peak in the scr*opr values, regarded as a function of the two position coordinates $\mathrm{x}$ and 
$y$, and checks to see whether this peak is long and narrow enough to be considered a line. Lines that do not pass through the "central" pixel of the local region defined by opr are rejected.

Note that positive $\mathrm{x}$ is to the right, positive $\mathrm{y}$ is downward, and positive angles are clockwise from the positive $x$ axis.

LIN02 bright, tangle, src, opr, length, width, avg

This is a line-finding operation like LIN01, except that LIN02 rejects lines if the absolute value of the average intensity in the local region is less than avg. LNN02 can find lines with negative brightness.

\section{LOG dst, src}

Set each image dst pixel equal to the natural logarithm of the corresponding image src pixel.

\section{MARK (no parameters)}

FOR PROGRAM G ONLY. Create or modify the 3-level mask to accompany the scene in image 1. The mask with its accompanying scene image (or images) is used in the training program F. This mask creation process is described in the user's manual.

MAX dst, src1, src2

Set each image dst pixel equal to the maximum of the corresponding image src1 pixel or the corresponding image src2 pixel.

\section{MAXCON dst, src, const}

Set each image dst pixel equal to the maximum of the corresponding image src pixel or the floating point value const.

\section{MEDIAN dst, src, opr [, Nlo [, Nhi ]]}

Do an order sort filter, in which the output (filtered) value is neither the largest nor the smallest of the values in the local region of the source image. This is a non-linear filter that removes local minima with domains of Nlo or fewer pixels, and local maxima with domains of Nhi or fewer pixels. For each pixel in image dst, MEDIAN finds the corresponding pixel in image src and aligns thereon the center pixel of operator opr. For each non-zero element of opr, the corresponding src pixel is put into a list of pixel values. The list is sorted according to value. The dst pixel value is set equal to the corresponding src pixel value, unless this value is smaller than the $(\mathrm{Nlo}+1)$ th smallest value or larger than the $(\mathrm{Nhi}+1)$ th largest value in the list, in which case the limiting list value is used for the dst pixel value. Thus, if Nlo is 1 and Nhi is 2, for example, the dst pixel value cannot be the smallest or the largest or the second largest value in the operator domain of src pixels. The operator values are not used except to specify which source pixels are in the local neighborhood, which is that part of the operator domain for which the operator values are not zero. src should have enough overlap rows to accommodate opr. Nlo and Nhi should normally be greater than 0 , and less than half of the number of non-zero operator elements. If either Nlo or Nhi is -1 , that -1 value is replaced by half (integer division by 2) of the number of non-zero elements in the operator. If Nlo is missing from the command, -1 is used for the missing value. If $\mathbf{N h i}$ is missing from the command line, the value of Nlo is used for the missing value. If the operator has an odd number of non-zero elements, and $\mathbf{N}$ is half of that number (integer division by 2 ), this 
MEDIAN operation is a standard median filter. Pixels in the excluded edge region of dst are set to zero. dst and src should be different images, and they may be different sizes.

Note that if Nlo is 0 and Nhi is one less than the number of non-zero elements in the operator, this MEDIAN operation gives the maximum of the pixel values in the local region; this and the similar $\mathrm{Nhi}=0, \mathbf{N l o}=\mathrm{N}-1$ are convenient ways to get a local maximum or local minimum. The code implements these special cases much more efficiently than the general case of MEDIAN.

\section{MED1X dst, src, $\mathbf{N}[$, xcl ]}

Set image dst equal to image src median filtered with a window of $\mathbf{N}$ pixels in the $\mathrm{X}$ (horizontal) direction by 1 pixel in the $y$ (vertical) direction. The domain of the operator xcl defines an excluded edge region, in which dst pixel values are left unchanged and src pixels are not used. If $\mathrm{xcl}$ is not given, 0 is used for its value. dst and src should be different images of the same size. $\mathbf{N}$ should be an odd integer.

MED1Y dst, src, $\mathbf{N}[$, xcl ]

Same as MED1X, except this filter is in the $y$ direction. This y-direction filter uses (N1)/2 overlap rows in src.

MIN dst, src1, src2

Set each image dst pixel equal to the minimum of the corresponding image src1 pixel or the corresponding image src2 pixel.

MINCON dst, src, const

Set each image dst pixel equal to the minimum of the corresponding image src pixel or the floating point value const.

MODMSK new, old, res, xcl, region, $\mathbf{Q}$

Set image new equal to a mask obtained by modifying the mask in image old. Image res is a result image from a previous program $\mathrm{E}$ calculation. If region is 2 , then any pixel with value 2 in old is changed to 0 in new if the res pixel value is less than $Q$. If region is 1 , then any pixel with value 1 in old is changed to 0 in new if the res pixel value is greater than $\mathbf{Q}$. If region is 3 , both operations are done. Other pixels are simply copied from old to new. Thus, the designated target (2) and background (1) regions in the mask are shrunk so that they do not extend beyond the target and background regions indicated in the result image res. This hopefully makes the mask more efficient without damaging its intended target and background designations. Operator xcl defines an excluded edge region in which new pixels are left unchanged. All three images should be the same size, and new may be the same image as either old or res.

\section{MOMUV src, opr, dst1 [, dstu [, dstv [, dstuu [, dstvv [, dsta ]1]1]}

MOMUV treats the intensity values in the local region as if they were an un-normalized probability density function (PDF), and calculates the second and lower moments of the PDF. MOMUV does a coordinate rotation to maximize the second moment about zero (not about the mean) in the $U$ direction. If the PDF indicates no preferred direction, the $U$ axis is along the $x$ axis (horizontal, positive to the right). Image src is the source image, and kernel opr defines the local region and the weights for the pixels in the local region. Images dst1, dstu, dstv, 
dstuu, dstvv and dsta are destination images for the weighted average intensity, the moments $\mathrm{U}, \mathrm{V}, \mathrm{UU}$, and VV (the UV moment is always 0 ), and the tangent of the angle from the $\mathrm{x}$ axis to the $\mathrm{U}$ axis. If the value 0 is used for any of the destination image numbers, the corresponding quantity is not written to any image. If an image number is not supplied for dstu ..., the value 0 is used for the missing image numbers. The $\mathrm{x}$ coordinate is positive to the right, and the $\mathrm{y}$ coordinate is positive downward, and the origin is at the center pixel of the local region. src must have enough overlap rows to accommodate opr. The images must all be the same size. src should normally be different from all the destination images. The excluded edge pixels in each dst are set to zero.

MUL dst, src1, src2

Set image dst $=$ image src1 $*$ image src2.

MULCON dst, src, const

Set image dst $=$ image src $*$ const.

\section{NEWIMG [(132 scalar variable)]}

Set $\$ \mathrm{~N}$ equal to the number of a not-yet-defined image. If all images are already defined, set $\$ \mathrm{~N}$ equal to -1 . If the name of an integer scalar variable is given as a parameter for this NEWIMG command, the variable's value is set equal to the $\$ \mathrm{~N}$ value.

\section{NLIN01 dst, bright, tangle, opr}

Count how many line extensions pass through each pixel. This operation is similar to XIIN01. But, whereas XLIN01 yeilds the sum of the intensities of all the line segments whose extensions would pass through the central pixel, NLIN01 is an attempt to count the number of line segments whose extensions would pass through the central pixel, independent of the line segment intensities. NLIN01 does not work very well. The weights in the operator opr should sum to 1.0 along any one ray from the center pixel.

\section{NTRP00 dst, src, opr}

NTRP00 does an interpolation, replacing pixels that have value 0.0 in the source image src with new values in the destination image dst. The new value is the weighted average of all the non-zero-value pixels in the local region, with the weights contained in the kernel opr. src should include enough overlap rows to accommodate opr. dst and src should be the same size. dst should usually be different from src.

\section{NTRP01 dst, src, opr}

NTRP01 does an interpolation, replacing pixels that have value 0.0 in the source image src with new values in the destination image dst. The new value is determined by a weighted least squares fit with a linear function of position to all the non-zero-value pixels in the local region, with the weights contained in the kernel opr. src should include enough overlap rows to accommodate opr. dst and src should be the same size. dst should usually be different from src. 
OUTM filename [, header ]

FOR PROGRAM G ONLY. Write the mask (image number 2) to file filename, optionally specifying the image file format with the parameter header which defaults to format 1. This is the same as the WRITEIMAGE command for image 2 .

\section{OUTS filename [, header ]}

FOR PROGRAM G ONLY. Write the scene image (image number 1) to file filename, optionally specifying the image file format with the parameter header which defaults to format 1. This is the same as the WRITEIMAGE command for image 1.

\section{OVERLAP img}

Set currently correct values in the overlap rows of image img. That is, each daisy obtains (from other daisies) the correct, current values of the pixels in its own overlap rows. This should be done before operations like CONVOLVE (convolution calculation) or MED1Y (median filter in the y direction) which use the overlap rows. The overlap rows are automatically assigned the correct values when an image is read from a file (commands READIMAGE and READSCENE). The overlap rows are NOT assigned the correct values for most other operations.

\section{PACC1 src, sum1, sumx, sumy, sumxx, sumyy, sumxy}

This command calculates sums for each src image peak previously defined by PEAK1 and PLNK1, which sums can be used to calculate the moments of each peak as if the peak were an un-normalized distribution function. On input, sum1 should be the same as peak output by PLNK1 or PMRG1, sumx should be accx, and sumy should be accy. This command treats each peak in src as a probability density function (unnormalized), calculates sums for each peak, and assigns the sum values to the destination image pixels corresponding to the accumulator pixel in peak (hence the term "accumulator"). sum1 is set equal to the sum of the sre pixel values in the peak. sumx and sumy are set equal to the sums of the distances (in pixels) from the accumulator pixel to the other pixels in the peak, multiplied by the src pixel value. sumxx, sumyy, and sumxy are set equal to the sums of the products of the distances, multiplied by src. The sums appear in the accumulator pixels only; the donor pixels are set to zero (except for src, which is unchanged). These images should all be the same size, and they should all be distinct. No overlap rows are needed for this operation (although some of the images need an overlap row for the preceding operations).

\section{PAUSE (no parameters)} struck.

The PAUSE command causes the program to wait until any key on the keyboard is

\section{PDFXYZ img [, xcl [, vmin, vmax ]]}

Treat the entire image img as a probability density function in two dimensions, and calculate certain moments. xcl is the number of a kernel that defines an excluded edge region. If only one parameter (img) is given for this PDFXYZ command, $x c 1$ is assumed to be 0 , which implies that no edge region is excluded. Only the pixels with values between vmin and vmax are included in this calculation. If fewer than 4 parameters are given with this PDFXYZ command, all pixels (except those in the excluded edge region) are included regardless of their values. This command sets $\$ A$ VGX $=$ average i value, $\$$ SIGX $=$ standard deviation in the $i$ 
direction, $\$ M I N I=$ minimum $i$ value, and $\$ M A X I=$ maximum $i$ value, and it sets the analogous user-accessible variables for the $j$ or $y$ direction. $i$ or $x$ increases from left to right, $j$ or $y$ increases from top to bottom, and $i=0, j=0$ is the top left pixel. This command also sets $\$ C O X Y=$ covariance of $i$ and $j$, and $\$ N=$ number of pixels included in the sums.

\section{PDFXY1 img, xcl, vmin, vmax}

This PDFXY1 command is similar to the PDFXYZ command, except that this PDFXY1 command uses 1.0 instead of the pixel value for the weight (the probability density function value) when calculating the weighted sums. This PDFXY1 command requires all 4 parameters.

\section{PEAK1 src, peak [, accx, accy ]}

This command finds peaks in image src. It compares each pixel in src with its 8 nearest neighbors. If the src pixel value is less than any of its neighbors, or if it is equal to a neighbor with a lower address (lower row number or same row and lower column number), the src pixel is declared not a peak and the corresponding pixel in peak is set to zero. Otherwise, the src pixel is considered a peak, and its value is assigned to the corresponding pixel in peak. This is intended to work with src images that have only non-negative pixel values. If values are given for parameters accx and accy, images accx and accy are set to zero for non-peak pixels, and they are set to $0.5+i$ and $0.5+j$ for peak pixels, where $i$ and $j$ are the peak pixel coordinates. The images accx and accy are set as an aid in the use of the PLNK1 command following this PEAK1 command. All the images should be distinct, and all should be the same size. src should have at least 1 overlap row. (The other images need overlap rows for later operations such as PLNK1.)

\section{PHEAD filename [, header [, length ]]}

Read and print the header from the image file filename. header is an integer code specifying the type of header (see READIMAGE). If the value -1 is given for header, or if no value is given for header, the program will attempt to determine the header type by itself. If the value 0 is given for header, the program simply prints the first length bytes of the file in hexadecimal and ASCII. The parameter length is not used, and need not be supplied, unless header is 0 .

\section{PLNK1 src, peak, accx, accy}

This command associates each non-zero pixel in image src with a nearby intensity peak. The PEAK1 command should be used to set the values in peak, accx, and accy, before this PLNK1 command is used. All four images should be the same size, all four should be distinct, and each of the four should have at least one overlap row.

For a peak in image stc, we will speak of an accumulator pixel and (usually) several donor pixels. The accumulator for a peak is the pixel with the greatest intensity in src in that peak; a donor is any pixel in that peak other than the accumulator (and with a src value greater than 0). The result of this PLNK1 operation is that each donor pixel in a peak is associated with the accumulator for that peak, by being assigned the accumulator pixel's values in images peak, accx, and accy. That is, for each donor pixel, the values of accx $-1 / 2$ and accy $-1 / 2$ are the coordinates of that donor's accumulator pixel, and the value of peak is the value of that donor's accumulator pixel in src. Each donor pixel is associated with the same peak as its nearest (of 8) neighbor pixel with the largest value in src. (This neighbor pixel value is larger than the donor's own value, or the "donor" is actually an accumulator.) If there is a tie for highest value 
nearest neighbor, the lower address neighbor is favored. This PLNK1 is intended for src images with non-negative pixel values. src pixels with value zero are not assigned to any peak. Unlike most operations, PLNK1 automatically sets the values in the overlap rows of flag, accx, and accy before those images are used, so you don't have to. You do have to set the (at least one) overlap rows in src, although usually the src overlap rows will be set before PEAK1 is used and they will still be set when PLNK1 is used.

PMOMUV sTc, mom0, avgu, avgv, varuu, varvv, tang, maxsag

PMOMUV is like PMOMXY with rotated coordinates, just as PMOMUV1 is like PMOMXY1 with rotated coordinates.

PMOMUV1 mom0, avgu, avgv, varuu, varvv, tang

This command is like PMOMXY1, in that it converts the sums from PACC1 into moments. However, this function works in a $\mathrm{u}-\mathrm{v}$ coordinate system which is rotated relative to the $x-y$ coordinate system, so that the largest second moment of the peak is along the $u$ axis. In this coordinate system, the second cross moment varuv is always zero. Instead of this moment, PMOMUV outputs the tangent of the angle from the $x$ axis to the $u$ axis, in the array tang. If the peak has no preferred direction, the $u$ axis is chosen along the $x$ axis. On input, mom0 should be the same as sum1 output by PACC1, avgu should be sumx, avgv should be sumy, varuu should be sumxx, varvv should be sumyy, and tang should be sumxy. These images should all be the same size, and they should all be distinct. No overlap rows are needed for this operation (although some of the images need an overlap row for the preceding operations).

PMOMXY src, mom0, avgx, avgy, varxx, varyy, varxy, maxsag

This PMOMXY function is simply a single command incorporating PEAK1, PLNK1, PMRG1, and PMOMXY1. This command treats each peak in image src as an un-normalized distribution function (probability density function), and calculates the moments of each peak. Two or more peaks connected by a path not lower than maxsag lower than the higher peak are treated as one peak. PMOMXY assigns the moment values to the destination image pixels corresponding to the maximum-value pixel in src, for each peak. mom0 is set equal to the sum of the src pixel values in the peak. avgx and avgy are set equal to the distance in pixels from the maximum-value pixel to the centroid of the peak. varxx, varyy, and varxy are set equal to the second moments (the variances and the covariance) about the centroid. These several moment values are put into the destination image pixels corresponding to the peak maximum pixel in src; the other destination pixels are set to zero. These images should all be the same size, and they should all be distinct, and they should all have at least one overlap row. Use the OVERLAP src command or some equivalent command before PMOMXY; OVERLAP is not necessary for the other images (PMOMXY does the other required overlap operations automatically).

\section{PMOMXY1 mom0, avgx, avgy, varxx, varyy, varxy}

This command converts the sums from PACC1 into moments. On input, mom0 should be the same as sum1 output by PACC1, avgx should be sumx, avgy should be sumy, varxx should be sumxx, varyy should be sumyy, and varxy should be sumxy. This command treats each peak in PACC1's src as a probability density function (unnormalized), calculates the moments for each peak, and assigns the moment values to the destination image pixels 
corresponding to the peak pixel in flag. mom0 is left unchanged, equal to sum1, the sum of the src pixel values in the peak. avgx and avgy are set equal to the distance in pixels from the accumulator (peak) pixel to the centroid of the peak. varxx, varyy, and varxy are set equal to the second moments (the variances and the covariance) about the centroid. These images should all be the same size, and they should all be distinct. No overlap rows are needed for this operation (although some of the images need an overlap row for the preceding operations).

\section{PMRG1 src, peak, accx, accy, sumxx, sumyy, sumxy, maxsag}

This command merges peaks that are connected by a path in src such that the lowest pixel value along that path is not lower than the higher peak value minus the (floating point) value maxsag and the lowest pixel is also greater than 0 . That is, all the pixels in two or more merged peaks are assigned to the same accumulator. The images src, peak, accx, and accy should be set by a prior call to the command PLNK1, and these four images will have the same meanings (although perhaps different values) after PMRG1 as after PLNK1. The images sumxx, sumyy, and sumxy are used for scratch by PMRG1. All seven images should have at least one overlap row, all should be the same size, and all should be distinct. It is not necessary for you to set the values of these overlap rows except for image src which is probably already set from the prior operations PLNK1 and PEAK1.

\section{PRINTS [src ...]}

Print the values of any scalar variables or file name variables listed as parameters. If no parameters are listed, all scalar variables are printed.

QUADUV src, opr, dst1 [, dstu [, dstv [, dstuu [, dstwv [, dsta ]]]]]

Like QUADXY, QUADUV fits the local region with a quadratic function. QUADUV then does a coordinate rotation from the $\mathrm{x}-\mathrm{y}$ to the $\mathrm{u}$-v coordinates, with the $\mathrm{u}$-axis chosen in the direction that maximizes the second derivative with respect to $u$. Image src is the source image, and kernel opr contains the weights for the pixels in the local region. Images dst1, dstu, dstv, dstuu, and dstvv are destination images for the coefficients of the $1, u, v, u u$, and vv terms in the fitted polynomial. (The uv term is always zero.) Image dsta is the destination for the tangent of the angle between the $x$ axis and the $u$ axis. If the value 0 is used for any of the destination image numbers, the corresponding quantity is not written to any image.

QUADXY src, opr, dst1 [, dstx [, dsty [, dstxx [, dstyy [, dstxy ]I]]]

QUADXY does a weighted least squares fit of a quadratic function of position to the pixels in a local region of the source image, and writes the 6 polynomial coefficients to the 6 destination images dst1, dstx, dsty, dstxx, dstyy, and dstxy. If the image number supplied for any destination image is 0 , the corresponding quantity is not written to any image. If an image number is not supplied for dstx ..., the value 0 is used for the missing image numbers. The $x$ coordinate is positive to the right, and the $y$ coordinate is positive downward, and the origin is at the center pixel of the local region. Image src is the source image. Kernel opr defines the local region and contains the weights. src must have enough overlap rows to accommodate opr. The images must all be the same size. src should normally be different from all the destination images. The excluded edge pixels in each dst are set to zero. 
READIMAGE filename, img [, header [, ncols [, nrow [, row0 [, col0 [, bpp ]11]1] (see also the following READIMAGE filename, -1 ... command)

Read all or part of an image from file filename into memory image img. The values of header indicate the type of header on the image file, as follows:

header meaning

0 . no header

1 Data Translation 512-byte header

2 Perceptron 11-byte header

3 Microsoft OS2 bit map file header

4 Microsoft Windows bit map file header

5 Daedalus 2048-byte header

6 Casi 1024-byte header

7 AMPS synthetic aperture radar

ncols is the number of columns in the file image. nrow is the number of rows of pixels to be read. The memory image img may be smaller than the file image in filename; the memory image is taken from that region of the file image with top left corner at row row0 and column col0. Thus, for the common case in which the file image is the same size as the memory image, row0 and colo should be set to 0 . bpp is the number of bytes per pixel. Acceptable values are $1 ; 2$, implying the less significant byte of each pair is first in the file; and -2 , implying two bytes per pixel with the more significant byte first. img must be greater than 0 .

Unlike most operations, READIMAGE does set the values of the pixels in the overlap rows of image img. If the file image does not have enough rows to fill the memory image, the unfilled memory image rows are left with the same values they had before.

The filename and img parameters must always be supplied with this command. If either of row0 or col0 is not supplied, it is assumed to be 0 . If any of header, ncols, nrow, or bpp is not specified, the value -1 is assumed. If any of these 4 parameters has the value -1 , the program attempts to determine a correct or reasonable value for the parameter from the file header, from the sizes of already-defined destination images, and from the BANDR parameters. This attempt may require the assumption of equal sizes for a file image and the corresponding memory image.

The image may be already defined, using DEFIMG, COPDEF, RESAMPLE, or a previous READIMAGE or READSCENE. If image img is not already defined, the program will attempt to define image img with ncols columns and nrow rows, with 0 overlap rows. If the header is type 1, the program will attempt to read the values of the scale factors $\mathbf{A}$ and $B$ from the header in the image file, and set the image table $A$ and $B$ values and the pre-defined scalar \$A and \$B values accordingly. Otherwise, these will be set to 0.0 and 1.0 .

READIMAGE filename, -1 [, header [, bpr [, nrow [, row0 ]1]] (see also the previous READIMAGE filename, img ... command)

This form of the READIMAGE command, with "-1" given instead of an image number, assumes that the file contains several images with interleaved rows. That is, the file contains the first row of the first image, the first row of the second image, ...., the first row of the Nth image, the second row of the first image, the second row of the second image, ..., perhaps with additional bytes interpsersed and perhaps with a file header. This command reads the several images from the file in one operation. Other than the following comments, this READIMAGE 1 command has the same features as the standard READIMAGE command. 
The information about each image or band is assumed to be already specified with the BANDR command. If any of the images specified in the BANDR command are not already defined when this READIMAGE -1 command is issued, this command will attempt to define those images. If nrow is given as -1 and the several images do not have the same number of rows, this READIMAGE -1 command will read only enough rows to fill the smallest destination image. bpr is the number of bytes in each composite row of the image file, including any row headers and any bands that are not of interest in this particular read operation. If bpr is given as -1 , the program will attempt to determine the correct value from the file header.

\section{READKERNEL filename, opr}

Read a local convolution operator (or kernel) from file filename into operator buffer number opr. The program assumes that the operator buffer has already been defined (operation DEFKERN) and that the buffer and the file are compatible. opr must be greater than 0 . The file is assumed to contain ASCII characters, with the several values separated by spaces, commas, or end-of-line characters.

\section{READSCENE img [, header [, ncols [, nrow [, row0 [, col0 [, bpp ]1]1]]]} READSCENE -1 [, header [, bpr [, nrow [, row0 ]1]] READSCENE (no parameters)

Read an image from the file whose name is in the scene image list file (.sl). This READSCENE is like READIMAGE, except that for READSCENE the file name is not given in the feature command file (.fc) but is given instead in the scene list file (.sl).

The program remembers previously specified names of scene files. If, instead of a file name, the entry in the scene list file (.sl) is a " + " followed by an integer value $n$, then the previously used file name for the $n$-th scene image is incremented and used as the present file name. (See the INCFIL command for an explanation of incrementing a file name.) If a " is given without an integer value, the value of $n$ is assumed to be the same as the number of the present scene image. For example, assume that this is the feature command file's third call to READSCENE (we are getting scene image 3), and that we are processing the fifth scene (this is the fifth use of the feature command file in this execution of the program). This call will use the third scene image file name in the fifth block of file names in the scene list file (.sl). If the file name is " + ", the file name used for the image 3 (the current scene image number) in scene 4 (the previous scene number) will be incremented and then used here. If the file name is " $+2^{\text {", }}$, the file name to be incremented and used here will be the one for image 2 of scene 5 , which is the last image 2 file name specified. If the file name is " $+4^{\prime \prime}$, the file name to be incremented and used here will be the one for image 4 of scene 4 , since the file for image 4 of scene 5 has not yet been specified.

A "." has an effect similar to a " + ", except that the old file name is not incremented before being used.

Each time the READSCENE command is used after the scene list file (.sl) is ended, the effect is the same as if " + " were read from the scene list file.

The scene list file (.sl) contains a block of image file names for each scene. For program $\mathrm{E}$, the first file name in each block is the name of the result file to be written, and the following file names in the block are real scene image file names numbered $1,2, \ldots$ For program $F$, there is no result image written, and the block of file names in the scene list file does not start with a result image file name. Instead, for program $F$, the file names in a block are all treated as scene image file names, and they are numbered $0,1,2, \ldots$ It is often convenient to use the 
mask image file name for the first scene image file name, so that the mask will be "scene image" number 0 and the "real" scene images will be numbered $1,2, \ldots$ Note that the first feature file written for each scene by program F must be the mask feature file, so it is handy if the first scene image file read is the scene mask image file.

The READSCENE command with no parameters reads and stores the next scene image file name from the scene list file (.sl) without reading any image. The specified file is not accessed at all; it does not even need to exist. This command is a way to get a file name into the program's memory without actually reading the file.

(READSCENE does not exist in program G.)

\section{READY (no parameters)}

Query all slaves, including the SCSI and video interfaces, and wait for each to.respond. This READY command causes the system to wait until all nodes have finished their tasks, which is useful for timing measurements and for debugging.

Usually, the fact that a slave responds to this or any other input indicates that the slave has finished previous tasks. However, if the slave is doing an operation that requires communication with other nodes, this READY query may cause an error condition in the slave program. Correctly written master node subroutines will prevent this query or any other communication from being sent to a slave while the slave is in such a vulnerable situation; the master node subroutine should not allow the input of any user command until all the slave nodes are no longer vulnerable.

REGISTER hhh, ggg, fff, fs1, gs1, gs2, gs3, gs4, bux, buy, bu1, bvx, bvy, bv1 [, dux [, duy [, du1 [, dvx [, dvy [, dv1 ]1]1]]

Register two images. That is, remap image ggg into image hhh so that the features in image hhh are optimally aligned with the corresponding features in image fff. Images ggg and fff are not changed by this REGISTER operation. Images fff and hhh must be the same size and must be compatible for arithmetic operations (they must be distributed the same way among the nodes); image ggg may be different in size. fs1, gs1, gs2, gs3, and gs4 are images that can be used as scratch space by this REGISTER operation. If 0 is given as the number for any of these scratch images, the program will find its own scratch space in place of that image (if sufficient memory is available). If a valid image number is given for any of these scratch images, then that image must be already defined. fs1 must be compatible with fff, and gs1, gs2, gs3, and gs4 must be compatible with ggg. All of the images must be distinct; none of the images needs any overlap rows. bux, buy, bu1, bvx, bvy, and bv1 are first guesses at the optimal mapping coefficients, as described for the REMAP operation using hhh for dst and using ggg for src. dux, duy, du1, dvx, dvy, and dv1 are the allowable ranges of variation of the mapping coefficients; that is, the final value of bux, for example, will not be allowed outside the range from bux-dux to bux +dux. If any of the range limits is not given, the value -1 will be used in its place. If a negative value is given (or assumed by default) for any of the range limits, the program will choose its own limit value. The final optimized values of bux, buy, bu1, bvx, bvy, and bv1 are returned in the user-accessible variables \$AVGX, \$AVGY, \$AVG, \$SIGX, \$SIGY, and \$SIG. The value of [1-corr] is returned in \$COXY, where corr is the crosscorrelation of the two images hhh and fff, with only the remapped ggg part of hhh and fff used if hhh is not completely covered by the remapped ggg. Possible corr values range from -1 to 1 , so $\$ \mathrm{COXY}=[1$-corr $]$ values range from 0 (perfect correlation) to 2 . 
REM (no parameters except a remark)

no action.

REMAP dst, src, bux, buy, bu1, bvx, bvy, bv1

Copy pixel values from image src to different pixels in image dst. The pixel location transformation is

$$
\begin{array}{ll}
(\text { src col }) & =\text { bux } *(\text { dst col })+\text { buy } * \text { (dst row })+ \text { bu1 } \\
(\text { src row }) & =\text { bvx } *(\text { dst col })+\text { bvy } * \text { (dst row) }+ \text { bv1 }
\end{array}
$$

where the coefficients are given as floating point values. If the corresponding src pixel does not exist, the dst pixel is left with the value it had before this operation. The overlap rows in dst are undefined after this operation.

\section{REPHI dst, src, const, rep}

Set image dst pixel equal to the corresponding image src if that src pixel value is no greater than const; otherwise, set the dst pixel value to the floating point value rep. (Values equal to const are not changed.)

\section{REPLO dst, src, const, rep}

Set image dst pixel equal to the corresponding image src if that src pixel value is greater than const; otherwise, set the dst pixel value to the floating point value rep. (Values equal to const are changed to rep.)

\section{RESAMPLE dst, src, xcl, ncol, nrow, novl}

Resample image src and put the result into image dst. The operator $x c l$ defines an excluded edge region of image sre; $x \mathrm{xl}$ may be 0 . There is no excluded edge in dst. If image dst is not yet defined, it will be defined with ncol columns, nrow rows, and novl overlap rows, and its scale factors $A$ and $B$ will be set to 0.0 and 1.0. Note that images defined by this RESAMPLE operation may be distributed among the daisies differently than images of the same size defined by other processes. If image dst was previously defined, it is assumed to have been defined with the correct size and with the correct parts allocated to each daisy, and the values supplied for ncol, nrow, and novl are ignored. The values of the overlap row pixels in dst are NOT set by this RESAMPLE operation. The resampling is done by the simplest nearest-pixel method, with no interpolation or smoothing. If image src has fewer rows than image dst and image src has overlap rows, the overlap rows will probably be used in the RESAMPLE procedure, so their values (if not already current) should be updated with the OVERLAP command before this RESAMPLE command is used. src overlap rows may also be used if the src and dst images are not distributed exactly correctly among the slave nodes, as might happen if one of the images is defined by a process other than a RESAMPLE of the other image.

\section{RETURN (no parameters)}

This command is the logical end of a subroutine. It causes the software to leave the subroutine and go to the command immediately following the GOSUB command that invoked the subroutine.

\section{RIJCON dst, src, opr}

This is an imperfect operation that tries to concentrate ridges. A weighted least squares fit is used to fit a quadratic polynomial to the local region in the source image src, with kernel 
opr containing the weights and specifying the local region. If the fitted polynomial describes a ridge, the ridge is smoothed along its length and concentrated in the direction perpendicular to its length, in the destination image dst. dst should be different from src, and they should be the same size. src should include enough overlap rows to accommodate opr. The excluded edge pixels in dst are set to zero.

SABS dst, src

Set scalar variable dst $=$ absolute value of src.

SADD dst, src1, src2

Set scalar variable dst $=$ src1 + src2. Or, concatenate two strings, which may be either file name variables or literal strings, and put the concatenated string in the file name variable dst.

SATAN dst, src

Set scalar variable dst $=$ arctangent of src, in radians from $-\mathrm{pi} / 2$ to $\mathrm{pi} / 2$.

SATAN2 dst, src1, src2

Set scalar variable dst $=$ arctangent of src1 / src2, in radians from - pi to pi.

SBEND dst [, src [, col0 [, addcol [, maxang]1]]

This SBEND operation does the s-bend geometric correction. For image src, the pixels within each row (or the columns in the image) are assumed to represent samples that are uniformly spaced in terms of the angle to the side of the camera center line. Image dst will have pixels that represent samples that are uniformly spaced in terms of the distance from the camera center line on a flat scene plane or a flat film plane. src and dst must be the same size, and they may be the same image. If a value is not given for src, it is assumed to be the same as dst. col0 is the (floating point) number of the image column corresponding to the camera center line, where angle $=0$. coll is the same for src and dst, and the src col0 pixels are mapped directly to dst colo. maxang is usually the maximum angle included in the camera's field of view, corresponding to pixels at the edge (largest column number) of the images, and column col0 + addcol is the pixel column number corresponding to this maximum angle. However, you are not required to use maxang and addcol values with this simple physical interpretation. Formally, the src pixels in columns colo + addcol and col0 - addcol are mapped directly to the same columns in dst. Pixels in columns other than these three special columns are shifted laterally (to different columns) in the SBEND operation. The columns numbered col0, col0 + addcol, and colo - addcol do not have to actually exist in the images src and dst.

If a value is not given for colo, it is assumed to be the center column in the images. If values are not given for addcol and maxang, they are assumed to be 357.0 columns and 0.75049 radian (43.0 degrees), which are the values for a normal Daedalus image.

\section{SCALE dst [, src [, xcl ]]}

Image dst is set equal to image src multiplied by a scale factor chosen to make the largest value in image dst equal to 254. The edge pixels, defined by operator xcl, are excluded; their values in src are not considered in determining the scale factor, and their values in dst are left unchanged. The image dst coefficient $A$ is set to 0.0 and $B$ is set to the scale factor used in this scaling operation, without regard to the coefficient values for image src, and the user-accessible variables $\$ A$ and $\$ B$ are set equal to $A$ and $B$. dst and src should be of the same size, and they 
may be the same image. If a value is not given for src, it is assumed to be the same as dst. Usually, SCALE should be used immediately before WRITEFEAT and other 8-bit image writing operations to be sure that the limited dynamic range of 8 bits per pixel is best utilized. SCALE is also useful before screen display operations.

\section{SDEFF32 src1 [src2 $\ldots$.}

Define 32-bit floating point variables with names src1, src2, ..., similar to SDEFI32.

\section{SDEFI32 src1 [src2 ...]}

Define 32-bit integer variables with names src1, src2, ... Variable names are limited to 30 characters. The first character of a variable name must be an alphabetic character, not a numeral or a special character. You must not try to re-define an already defined variable. This operation does not assign values to the newly-defined variables. If a variable name is immediately followed by brackets enclosing an integer constant or an already-defined integer variable (with no intervening spaces), the variable is defined as an array instead of a scalar. For example, the command

\section{SDEFI32 AAA[7] B}

defines $B$ as a single type $\mathrm{I} 32$ (32-bit integer) variable and AAA as an array of 7 type $\mathrm{I} 32$ variables which can be referred to individually as AAA[0], AAA[1], ... AAA[6].

SDIV dst, src1, src2

Set scalar variable dst $=\mathbf{s r c 1} /$ src2.

\section{SEGLAB dst, src}

SEGLAB assigns unique labels to image segments. SEGLAB assumes that image src has patches of marked pixels, with values greater than 0 , separated by regions of background pixels, with values less than or equal to 0 . For each marked src pixel, SEGLAB sets the corresponding destination image dst pixel to some value that comprises a label for the segment. Each contiguous segment gets a unique label, equal to $1+r+c / n c$ where $n c$ is the number of columns in the images and $\mathrm{r}$ and $\mathrm{c}$ are the row and column number for the lowest-numbered pixel in the segment. dst pixel values are not changed if the corresponding src pixels are not marked as segments. Normally, all dst pixels should be set to 0 before starting a segmentation procedure that uses this subroutine. dst pixel values that are not 0 on entering this subroutine are construed as indicating a pixel that is already labeled. A non-zero value for a dst pixel and a greater-than-zero value for the corresponding src pixel is an inconsistency, indicating overlapping patches (segments). When this occurs, the old labels will not be changed, and the non-overlapping parts of the new overlapping patches will get a new label. Successive uses of the same destination image with different source image will result in unique labels for all the patches (segments) in the several source images as long as those patches do not overlap. src and dst must be different images, they must be of the same size, and both dst and src must have at least one overlap row.

SEQ dst, src

Set (numerical, integer or floating) scalar variable dst $=\mathbf{s r c}$, where src may be either a scalar variable or a numerical constant. Or, set file name variable dst equal to src, where src may be either a file name variable or a literal string with no embedded spaces. 
SET_AB img, A, B

$S E T A B$ img

The first form sets the $A$ and $B$ coefficients for image img to floating point values $A$ and B. ([This image] $=A+B *$ [original image].) These coefficients were initially set to $A=0.0$, $B=1.0$ when the image was defined. This operation does NOT rescale the image; it merely sets the values of the stored coefficients. The second form does not change the A and B coefficients for image img. Both forms then set the user-accessible variables $\$ A$ and $\$ B$ equal to the $A$ and $B$ values for image img.

\section{SETPIX img, row, col, val}

Set the pixel in image img, row row, column col, to the floating point value val. This operation sets pixels in both the primary and the overlap rows. Note that images are stored in memory as floating point data.

SEXP dst, src

Set scalar variable dst $=$ exponential (inverse natural logarithm) of src.

SLOG dst, sTc

Set scalar dst = natural logarithm of src.

SMAX dst, src1, src2

Set scalar variable dst $=$ maximum of src1 or src2.

SMIN dst, src1, src2

Set scalar variable dst $=$ minimum of src1 or src2.

\section{SMTHX dst, src, sigma, $\mathbf{N}[$, xcl ]}

Set image dst equal to image src smoothed in the $x$ (horizontal) direction. The smoothing operation comprises $\mathbf{N}$ repetitions of a two-pass (one pass increasing $\mathrm{x}$, one pass decreasing $x$ ) exponential smoothing filter, with the exponential filter width chosen to make the half-width (the standard deviation) of the smoothing function of the total operation equal to the floating point value sigma. Larger $\mathbf{N}$ values make the overall smoothing function look more Gaussian. The domain of the operator xcl specifies an excluded edge region, in which the dst pixel values are not changed. (The operator xcl is not used for anything except specifying this excluded edge region.) dst should be the same size as src, and they may be the same image.

SMTHXS dst, src, sig [, xcl ]

Set image dst equal to image sre smoothed in the $x$ (horizontal) direction. The smoothing operation comprises a two-pass (one pass increasing $x$, one pass decreasing $x$ ) exponential smoothing filter. The filter width is not necessarily constant for the whole image area; the exponential filter width for each pixel is the value of the corresponding pixel in the image sig. Such a smoothing operation, with the smoothing width varying from one pixel to the next, might not have some properties normally expected from smoothing operations, such as conservation of the area (or volume) under a peak. The domain of the operator xcl specifies an excluded edge region, in which the dst pixel values are not changed. (The operator xcl is not used for anything except specifying this excluded edge region.) dst, src, and sig should all be 
the same size. dst and src may be the same image, but sig should be different from both dst and src.

SMTHY dst, src, sigma, N [, xcl ]

Same as SMTHX, but this smoothing is in the $y$ (vertical) direction. (Overlap rows are not needed.)

SMTHYS dst, stc, sig [, xcl ] not needed.)

Same as SMTHXS, but this smoothing is in the y (vertical) direction. (Overlap rows are

SMUL dst, src1, src2

Set scalar variable dst $=\mathbf{s r c 1} * \mathbf{s r c 2}$.

SQRT dst, src

Set image dst $=$ square root of image src.

SSQ dst, src, opr

Set image dst equal to the local sum of squares of image src, with weighting coefficients from operator opr. That is, set each image dst pixel value equal to the sum over the domain of operator opr of $\left\{\mathrm{src}^{*} \mathrm{Src}^{*} \mathrm{opr}\right\}$. The dst pixels in the excluded edge region, defined by the domain of the operator opr, are set to zero. src should have enough overlap rows to accommodate operator opr. opr must be greater than 0 . dst and src should be different images. The image dst does not need to be the same size as the image src, but the two images do need to be distributed among the daisies in a manner compatible with the RESAMPLE algorithms.

SSQRT dst, src

Set scalar variable dst $=$ square root of src.

SSUB dst, src1, src2

Set scalar variable dst $=\mathbf{s r c 1}-\mathbf{s r c 2}$.

STAN dst, sTc

Set scalar variable dst $=$ tangent root of src, with src in radians.

\section{STATS img [, xcl ]}

Calculate pixel value statistics for image img, excluding the edge region defined by the domain of operator xcl. If $x c 1$ is not given, the value 0 is assumed and no edge region is excluded. This operation sets the user-accessible variables $\$ \mathbf{N}=$ number of pixels, $\$ A V G=$ average value of pixels, $\$$ SIG $=$ standard deviation of pixel values, $\$ M A X=$ maximum pixel value, $\$ M M N=$ minimum pixel value, $\$ N R O W=$ number of rows, $\$ N C O L=$ number of columns, $\$ \mathrm{~A}=$ value of $\mathrm{A}$ coefficient in tables, $\$ \mathrm{~B}=$ value of $\mathrm{B}$ coefficient in tables.

STOP (no parameters)

Stop the feature calculation process for the current scene. For program G, stop execution of the whole program. For program $E$, go on to the next scene. For program F, accumulate 
the sums for this scene and then go on to the next scene, optimizing the coefficients after the last scene.

SUB dst, src1, sTc2

Set image dst = image src1 - image src2.

SUBCON dst, src, const

Set image dst $=$ image src - const.

SUBDEF subname [typcod, varnam] $[, \ldots]$

Define a "subroutine" in a .fc file. This is the first line of a subroutine in a .fc file. A subroutine is a block of commands that can be executed from elsewhere in the .fc file via the GOSUB command. The subroutine starts with this SUBDEF command, normally includes several other commands, and normally ends with the RETURN command which transfers control back to the line following the GOSUB command that invoked the subroutine. (Actually, the subroutine does not necessarily need a RETURN as its last physical line in the .fc file, but the logical end of a subroutine must be a RETURN.) The subroutine, and hence the SUBDEF command, should be placed in the .fc file in such a way that the SUBDEF command is never encountered except via the GOSUB command, and the RETURN command is never encountered except after a SUBDEF command.

The subroutine name subname is treated as a user-defined variable. Thus, it can be chosen more or less arbitrarily within the limits of normal variable names, and it must be distinct from all other variable names.

Each subroutine variable is specified by two parameters in the SUBDEF command. The first parameter of the pair specifies the type of variable: I32 (32-bit integer), F32 (32-bit floating point), CHR (character), or STR (character string). The second parameter of the pair is the name of the subroutine variable. The name must conform to the rules for user-defined variable names. It must be distinct from all other variable names in the .fc file, and it must not be defined anywhere else in the .fc file. That is, all variables, both within a subroutine and outside of any subroutine, are "global" in the sense that their names are all kept in the same list and they are all accessible from anywhere in the entire .fc file. Subroutine variables are not in any way isolated from other variables.

A subroutine variable can be either a scalar or a one-dimensional array. To specify that a subroutine variable is an array, the name of the variable in the SUBDEF command is immediately followed by an empty bracket pair " $\mathrm{C}$ ". Thus, the command line

SUBDEF ABC I32 XXXП 132 Y STR Z

specifies that $A B C$ is the subroutine name; $X X X$ represents an array of $I 32$ values within the subroutine; $\mathrm{Y}$ represents a single $\mathrm{I} 32$ value within the subroutine; and $\mathrm{Z}$ represents a character string within the subroutine. Within the subroutine, we can refer to individual elements of the array $\mathrm{XXX}$, with commands like

SADD XXX[ग XXX[2] Y

where $J$ is a variable that is defined elsewhere in the .fc file. This command sets element number $\mathrm{J}$ of array $\mathrm{XXX}$ equal to the sum of $\mathrm{Y}$ and element number 2 of $\mathrm{XXX}$.

The subroutine variables are accessible from elsewhere in the .fc file. However, you must use caution and not access these variables until after they have been defined, which occurs when the subroutine is defined. This happens the first time the software scans over the SUBDEF 
command in the .fc file, even if the software is merely jumping over the SUBDEF command in response to a JUMP command or a GOSUB to a different subroutine.

\section{SUMPIX img [, xcl ]}

Calculate the sum of all the pixels in the image img, excluding the edge region defined by the domain of operator xcl. If $x c 1$ is not given, the value 0 is assumed and no edge region is excluded. This operation sets the user-accessible variables $\$ N=$ number of pixels, $\$ A V G=$ average value of pixels.

\section{TAN dst, src}

Set each image dst pixel equal to the tangent of the angle specified by the value of the corresponding image src pixel. Angles must be in radians.

\section{UNDERSAMPLE dst, src, xcl, xfact, yfact, novl}

Undersample image src and put the result into image dst. This function is the same as RESAMPLE, except that, whereas RESAMPLE specifies the size of the new image dst directly, UNDERSAMPLE uses xfact and yfact to specify the size of the new image dst relative to the size of the old image src. The size of the new image dst will be the size of the old image src minus the excluded edge regions defined by the domain of the operator xcl, divided by $x$ fact in the horizontal direction and yfact in the vertical direction. Images defined by this UNDERSAMPLE operation are completely compatible with (are distributed among the slave nodes the same way as) images defined by the RESAMPLE operation.

\section{VGA caption, img [, row0 [, col0 [, Q ]]]}

Display image img on the system (console) monitor. The top left corner of the image will appear at row0, col0 in the screen display. Red will be used for pixels with value less than $\mathbf{Q}$, green for pixels with value greater than $\mathbf{Q}$. The default values for row0, col0, and $\mathbf{Q}$ are 0,0 , and 0.0 . Pixel values should be between 0.0 and 255.0. The character string caption will be printed below the image on the screen. caption should not contain any embedded spaces. If $a$ "." is the first character of the string given as the label, then the last-used result file name will be used as the label. This requires that Gordon Lassahn's device handler VGA and compatible video hardware are installed. This display function is rather crude, slow, and buggy, and is not generally recommended.

\section{WRITEFEAT filename, sTc}

FOR PROGRAM F ONLY. Write image src to file filename, as in operation WRITEIMAGE, and enter filename into an internal list of feature image files. If a string beginning with " + " is given instead of a file name, the previously used feature image file name is incremented and used here. (See the INCFL command for an explanation of incrementing a file name.) The initial stored feature image file name is LFIIFI000000.FI for ATR1 and ATR2, and /usr/users/fi/fi000000.fi for ATR3. Feature images are always written with a Data Translation header, in which is embedded the value of the last scale factor B used in scaling the image with the SCALE command.

Feature image files are written with each pixel represented as one 8-bit (1-byte) integer. This means that any pixel value outside the range 0.0 to 255.0 will not be correctly represented. It also means that precision is limited. As a worst case example, if the feature image pixel values range from 0.0032 to 0.4158 , they will all be written as 0 and all precision will be lost. 
You must construct feature images in such a way that there are no negative values. After this is done, you should usually use the SCALE command just before the WRITEFEAT command. This scale command multiplies the image by a constant that allows optimal use of the available precision, and this constant is written to the feature image file and it is used later by the $F$ program to restore the feature image pixels to their original (before scaling) values during the sums calculations.

\section{WRITEIMAGE filename, src [, header ]}

Write image number src to file filename. If the value of header is 0 , no header is written to the file; if the value is 1 , a Data Translation header is written. If header is not given, a value of 1 is used. Bytes 127 through 152 of the Data Translation header (the first byte of the header is called number 1) will be the scale coefficients A and B in FORTRAN format $(1 \mathrm{x}, \mathrm{e} 12.6,1 \mathrm{x}, \mathrm{e} 12.6)$. No other values can be used for header with WRITEIMAGE.

\section{WRITERESULT sTc, header, $\mathbf{Q}$}

FOR PROGRAM E ONLY. This is the same as command WRITEIMAGE, with two additional features. If header is 1 , the value $\mathbf{Q}$ is stored in the file header in place of the value of the scale factor A. The file name for this result image output file is read from the scene list file (.sl), the first of the set of file names given for the scene, the name that takes the place of the name of the mask image in the scene list file used for the training process. If " + " is given instead of a file name in the scene list file (.sl), then the previously used result image file name is incremented and used here. The initial file name is SCSIDISK:Irilri000000.ri for ATR1, Irilri000000.ri for ATR2, and /usr/users/ri/ri000000.ri for ATR3.

\section{XGRAD dst, src}

Set image dst equal to the $\mathrm{x}$-gradient (derivative with respect to $\mathrm{x}$, the coordinate that increases from left to right) of the intensity in image src. The gradient values are calculated as gradient $=$ (intensity $[i+1]$ - intensity [i-1]) / 2. The first and last columns (left and right edges) of dst are set to 0 . dst and src should be different images, of the same size.

\section{XIMG dst}

Set the value of each pixel in image dst equal to the pixel's $x$ (column) coordinate.

\section{XLIN01 dst, bright, tangle, opr}

Extend lines found by LIN01. The "central" pixel in the local region of image dst is set equal to the weighted sum of the brightnesses of the line segments that are in the local region defined by the operator opr and are oriented in the direction so that their extensions would pass through the central pixel. The weights are the values of the pixels in opr. bright and tangle are the images containing the brightness and orientation data from LIN01. dst, bright, and tangle should all be different images, all of the same size. This operation uses overlap rows for both images bright and tangle. dst pixels in the excluded edge region defined by the domain of opr are set to zero.

\section{XY2RT dstr, dstt, srcx, srcy [, xcl ]}

Convert a vector field from $x-y$ representation to $r$-tangent(angle) representation. Images srcx and srcy contain the $x$ and $y$ component values. The vector magnitude will be put into image dstr, and the tangent of the angle between the vector and the $x$ axis will be put into image 


\section{DISCLAIMER}

This report was prepared as an account of work sponsored by an agency of the United States Government. Neither the United States Government nor any agency thereof, nor any of their employees, makes any warranty, express or implied, or assumes any legal liability or responsibility for the accuracy, completeness, or usefulness of any information, apparatus, product or process disclosed, or represents that its use would not infringe privately owned rights. References herein to any specific commercial product, process, or service by trade name, trademark, manufacturer, or otherwise, does not necessarily constitute or imply its endorsement, recommendation, or favoring by the United States Government or any agency thereof. The views and opinions of authors expressed herein do not necessarily state or reflect those of the United States Government or any agency thereof. 